\title{
2,3-二取代喹唑啉-4(3H)-酮化合物的合成及生物活性研究
}

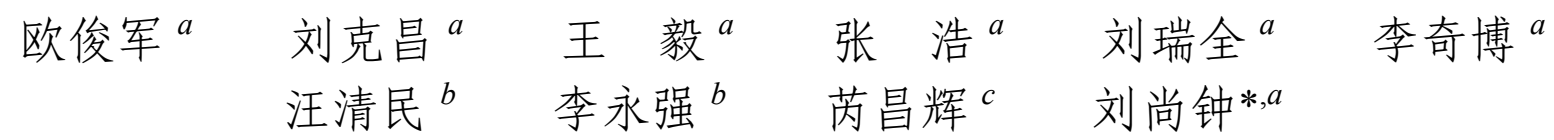

$\left({ }^{a}\right.$ 中国农业大学理学院 应用化学系 北京 100193)

( ${ }^{b}$ 南开大学元素有机化学国家重点实验室 天津 300071)

( ${ }^{c}$ 中国农业科学研究院植物保护研究所 北京 100193)

\begin{abstract}
摘要 设计合成了一系列 2,3-二取代喹唑啉-4(3H)-酮化合物, 并研究了常用无机干燥剂对目标化合物合成的影响, 发 现无水硫酸镁能够促进 2,3-二取代喹唑啉-4(3H)-酮化合物的形成. 所有化合物通过核磁共振氢谱、核磁共振碳谱、高 分辨质谱等方法进行结构表征，并首次报道了 6-氯-3-芐氨基-2-新戊基-8-甲基喹唑啉-4(3H)-酮的单晶结构. 通过生物 活性测试发现部分化合物在 $200 \mathrm{mg} / \mathrm{L}$ 浓度下对小麦白粉病(Blumeria graminis)具有较好的活体防效.

关键词 2,3-二取代喹唑啉-4(3H)-酮; 合成; 生物活性; 单晶结构
\end{abstract}

\section{Synthesis and Bioactivity Studies of 2,3-Disubstituted Quinazolin-4(3H)-one}

\author{
Ou, Junjun ${ }^{a}$ \\ Liu, Kechang ${ }^{a} \quad$ Wang, $\mathrm{Yi}^{a}$ \\ Zhang, $\mathrm{Hao}^{a}$ \\ Liu, Ruiquan ${ }^{a}$ \\ $\mathrm{Li}, \mathrm{Qibo}^{a}$ \\ Wang, Qingmin ${ }^{b}$ \\ Li, Yongqiang ${ }^{b}$ \\ Rui, Changhui ${ }^{c}$ \\ Liu, Shangzhong*,a \\ ( ${ }^{a}$ Department of Applied Chemistry, College of Science, China Agricultural University, Beijing 100193) \\ ( ${ }^{b}$ State Key Laboratory of Elemento-Organic Chemistry, Nankai University, Tianjin 300071) \\ ( ${ }^{c}$ Institute of Plant Protection, Chinese Academy of Agricultural Sciences, Beijing 100193)
}

\begin{abstract}
In this research, a series of new 2,3-disubstituted quinazolin-4(3H)-ones were designed and synthesized. The influence of common drying agents on the formation of 2,3-disubstituted quinazolin-4(3H)-ones was investigated and firstly reported. It is found that the anhydrous magnesium sulfate can increase the yield of 2,3-disubstituted quinazolin-4(3H)-ones significantly. The structures of new compounds were all confirmed via ${ }^{1} \mathrm{H}$ NMR, ${ }^{13} \mathrm{C}$ NMR and HRMS. At the same time, the crystal structure of 3-(benzylamino)-6-chloro-8-methyl-2-neopentyl-quinazolin-4(3H)-one was firstly reported. The results of bioactivity assay showed that some of these new compounds have some control effects on wheat powdery mildew (Blumeria graminis) in vivo at a concentration of $200 \mathrm{mg} / \mathrm{L}$.
\end{abstract}

Keywords 2,3-disubstituted quinazolin-4(3H)-ones; synthesis; bioactivities; crystal structure

结构多变性和活性多样性的喹唑啉酮类化合物在 医药的抗癌 ${ }^{[1]}$ 、抗菌 ${ }^{[2]}$ 、抗炎 ${ }^{[3]}$ 、抗病毒 ${ }^{[4]}$ 、抗惊厥 ${ }^{[5]}$ 、 降血压 ${ }^{[6]}$ 等方面都具有良好的生物活性, 例如目前在临 床上使用的抗癌药物吉非替尼(Gefitinib) ${ }^{[7]}$ 和厄洛替尼 (Erlotinib $)^{[8]}$ 都具有喹唑啉酮的结构; 一些结构表现出杀 螨、杀菌农药活性, 如已经商业化的喹唑啉酮类农药有 氟喹唑(Fluquinconazole)、灭螨猛(Chinomethionate) 等 $^{[9]}$.
鉴于该类结构具有优异的生物活性，近年许多课题组研 究该类化合物新的农药活性，发现不同结构的化合物分 别具有较好抗病毒活性 ${ }^{[10]}$ 、杀虫 ${ }^{[1]}$ 和杀菌活性 ${ }^{[12]}$.

随着微波、超声波、金属催化剂等在有机合成中的 应用，促进了喹唑啉酮类化合物的合成和相应活性化合 物的发现 ${ }^{[13]}$. 文献中报道的喹唑啉酮类化合物的合成 多以邻氨基苯甲酰胺、靛红酸䣶、邻氨基苯甲酸、邻卤

*E-mail: shangzho@cau.edu.cn

Received October 16, 2013; revised November 13, 2013; published online November 21, 2013.

Project supported by the National Natural Science Foundation of China (Nos. 20972186, 21172256), the National Basic Research Program of China (No. 2010CB126104) and the Project of Green Pesticide Research, Development and Industrialization (No. 2011BAE06B03).

国家自然科学基金(Nos. 20972186, 21172256)、国家重点基础研究发展计划(No. 2010CB126104)和绿色生态农药的研发及产业化(No. 2011BAE06B03) 资助项目. 
苯甲酸、邻氨基苯甲腈、邻硝基苯甲腈等 ${ }^{[14]}$ 为起始原料, 在以靛红酸䣶为原料合成喹唑啉酮类化合物的文献中, 为了提高原料转化率及底物扩展性, 反应中加入了金属 催化剂(三氟甲磺酸镓、三氟甲磺酸锡等)或脱水剂(硫 酸、硝酸铈铵等)促进喹唑啉酩环 ${ }^{[15]}$ 的形成.

我们课题组设计了以取代靛红酸酤为原料, 通过酰 基化、开环及环化反应合成了一系列 2,3-二取代喹唑 啉-4(3H)-䣶类化合物, 旨在发现新型的杀菌活性化合 物. 在目标产物的合成反应中, 研究了无机干燥剂对反 应的影响, 发现在反应体系中加入一定量的无水硫酸 镁, 可以促进目标产物的生成. 同时, 对所有合成的化 合物进行了抗肿瘤、杀虫、杀菌等生物活性的测试, 发 现合成的系列化合物具有一定的杀菌活性. 化合物结构 信息见表 1 , 合成路线见 Scheme 1.

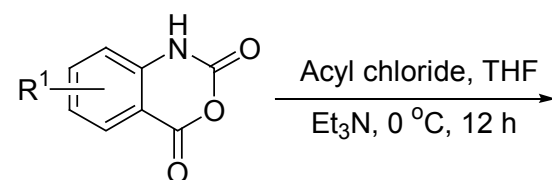

A<smiles>[R][M]1ccc2nc([R])oc(=O)c2c1</smiles>

M
Benzylamine, THF $\mathrm{MgSO}_{4}$, reflux, $3 \sim 5 \mathrm{~h}$<smiles>[R]Cn1c([R])nc2c(c1=O)C=[X][R]C=C2</smiles>

Scheme 1

\section{1 结果与讨论}

\section{1 合成}

反应中间体需要脱去一分子水转化为目标产物 $\mathbf{Q}$,
通常情况下，选用合适溶剂通过共沸脱水及时将生成的 水从反应体系中移走，可以促进目标产物的生成和提高 反应产率. 然而有些反应由于溶剂或者反应温度的限 制, 无法采用共沸蒸馏. 本文利用无机干燥剂的强吸水 作用, 尝试研究了四种常规干燥剂对由 2-丁基- $4 H$-苯并 $[d][1,3]$ 噁嗪-4-酤与 2-氨甲基吡啶合成 2-丁基-3-(吡啶 -2-基甲基)喹唑啉-4(3H)-酮(Q-01)的影响, 发现 4 种干 燥剂皆能不同程度提高反应产率(表 2 ).

其中以无水硫酸镁为脱水剂时，可将产率由 $49 \%$ 提 高至 $87 \%$. 而无水氯化钲和无水硫酸钠对产率提高较 小, 产率仅分别提高至 $65 \%$ 和 $56 \%$. 可能的原因是氯化 钻在 $40{ }^{\circ} \mathrm{C}$ 左右能溶解在 0.6 倍量的水中, 而十水硫酸 钠在 $32.4{ }^{\circ} \mathrm{C}$ 时容易失去全部结晶水 ${ }^{[16]}$, 二者在高温下 均无法形成稳定的结晶水合物而达到脱水目的. 此外, 以无水硫酸铜为脱水剂时，产率虽然达到了 $73 \%$, 但在 反应过程中茮胺的用量需要增加到 3 倍. 原因在于铜离 子在加热条件下易与芐胺先形成稳定络合物, 而影响芐 胺参与反应.

本文中反应用四氢呋喃作溶剂, 以无水硫酸镁为脱 水剂, 反应结束后只需要将反应液过滤后, 在滤液中加 入一定量的石油醚进行结晶就能得到产物. 同文献[17] 报道的类似方法用甲苯或 $N, N$-二甲基甲酰胺作溶剂、对 甲苯磺酸作催化剂相比，操作容易、后处理简单、收率 较高.

文献[18]报道的 3-苠氨基取代喹唑啉酮化合物的合 成方法主要是通过水合肼与苯并啞嗪酮反应生成 3-氨 基取代喹唑啉酮，与芳香醛反应生成亚胺后氢化还原得 3-芐氨基取代喹唑啉酮. 该反应路线长, 总收率不高.

表 1 化合物 Q-01 Q-31 一览表

Table 1 The structure information of compounds $\mathbf{Q - 0 1} \sim \mathbf{Q - 3 1}$

\begin{tabular}{|c|c|c|c|c|c|c|c|}
\hline Compd. & $\mathrm{R}^{1}$ & $\mathrm{R}^{2}$ & $\mathrm{R}^{3}$ & Compd. & $\mathrm{R}^{1}$ & $\mathrm{R}^{2}$ & $\mathrm{R}^{3}$ \\
\hline Q-01 & $\mathrm{H}$ & $n$-Butyl & 2-Pyridyl & Q-17 & $\mathrm{H}$ & Cyclopropyl & $2-\mathrm{FC}_{6} \mathrm{H}_{4}$ \\
\hline Q-02 & $\mathrm{H}$ & $n$-Butyl & $4-\mathrm{ClC}_{6} \mathrm{H}_{4}$ & Q-18 & $\mathrm{H}$ & $n$-Pentyl & $3-\mathrm{ClC}_{6} \mathrm{H}_{4}$ \\
\hline Q-03 & $\mathrm{H}$ & $n$-Butyl & $3-\mathrm{BrC}_{6} \mathrm{H}_{4}$ & Q-19 & $\mathrm{H}$ & $n$-Pentyl & $4-\mathrm{FC}_{6} \mathrm{H}_{4}$ \\
\hline Q-04 & $\mathrm{H}$ & n-Butyl & $4-\mathrm{BrC}_{6} \mathrm{H}_{4}$ & Q-20 & $\mathrm{H}$ & $n$-Pentyl & $3-\mathrm{FC}_{6} \mathrm{H}_{4}$ \\
\hline Q-05 & $\mathrm{H}$ & $n$-Butyl & 4- $\mathrm{CH}_{3} \mathrm{C}_{6} \mathrm{H}_{4}$ & Q-21 & $\mathrm{H}$ & $n$-Pentyl & $2-\mathrm{FC}_{6} \mathrm{H}_{4}$ \\
\hline Q-06 & $\mathrm{H}$ & n-Butyl & $2-\mathrm{CH}_{3} \mathrm{C}_{6} \mathrm{H}_{4}$ & Q-22 & $\mathrm{H}$ & $n$-Butyl & $3-\mathrm{ClC}_{6} \mathrm{H}_{4}$ \\
\hline Q-07 & $\mathrm{H}$ & $n$-Butyl & $3-\mathrm{OCH}_{3} \mathrm{C}_{6} \mathrm{H}_{4}$ & Q-23 & $\mathrm{H}$ & $n$-Butyl & $4-\mathrm{FC}_{6} \mathrm{H}_{4}$ \\
\hline Q-08 & $\mathrm{H}$ & $n$-Butyl & $4-\mathrm{OCH}_{3} \mathrm{C}_{6} \mathrm{H}_{4}$ & Q-24 & $\mathrm{H}$ & $n$-Butyl & $3-\mathrm{FC}_{6} \mathrm{H}_{4}$ \\
\hline Q-09 & $\mathrm{H}$ & $n$-Butyl & $\mathrm{C}_{6} \mathrm{H}_{5}$ & Q-25 & $\mathrm{H}$ & $n$-Butyl & $2-\mathrm{FC}_{6} \mathrm{H}_{4}$ \\
\hline Q-10 & $\mathrm{H}$ & $n$-Butyl & 4-tert-Butyl- $\mathrm{C}_{6} \mathrm{H}_{4}$ & Q-26 & $8-\mathrm{CH}_{3}$ & $n$-Butyl & $3-\mathrm{ClC}_{6} \mathrm{H}_{4}$ \\
\hline Q-11 & $\mathrm{H}$ & $n$-Butyl & 3-Pyridyl & Q-27 & $8-\mathrm{CH}_{3}$ & $n$-Butyl & 4- $\mathrm{FC}_{6} \mathrm{H}_{4}$ \\
\hline Q-12 & $\mathrm{H}$ & Cyclopropyl & $4-\mathrm{FC}_{6} \mathrm{H}_{4}$ & Q-28 & $8-\mathrm{CH}_{3}$ & $n$-Butyl & $3-\mathrm{FC}_{6} \mathrm{H}_{4}$ \\
\hline Q-13 & $\mathrm{H}$ & Cyclopropyl & $4-\mathrm{OCH}_{3} \mathrm{C}_{6} \mathrm{H}_{4}$ & Q-29 & $6-\mathrm{Cl}$ & Cyclohexyl & $4-\mathrm{FC}_{6} \mathrm{H}_{4}$ \\
\hline Q-14 & $\mathrm{H}$ & Cyclopropyl & 4- $\mathrm{ClC}_{6} \mathrm{H}_{4}$ & Q-30 & $6-\mathrm{Cl}$ & Cyclohexyl & $2-\mathrm{FC}_{6} \mathrm{H}_{4}$ \\
\hline Q-15 & $\mathrm{H}$ & Cyclopropyl & $4-\mathrm{BrC}_{6} \mathrm{H}_{4}$ & Q-31 & $6-\mathrm{Cl}$ & Cyclohexyl & $3-\mathrm{FC}_{6} \mathrm{H}_{4}$ \\
\hline Q-16 & $\mathrm{H}$ & Cyclopropyl & 4-tert-Butyl- $\mathrm{C}_{6} \mathrm{H}_{4}$ & & & & \\
\hline
\end{tabular}


表 2 脱水剂的篮选

Table 2 Screening of dehydrate agents

\begin{tabular}{ccc}
\hline 脱水剂 $^{a}$ & 反应时间 $/ \mathrm{h}$ & 产率 ${ }^{b} / \%$ \\
\hline 无 & 12 & 49 \\
无水氯化钻 & 12 & 65 \\
无水硫酸钠 & 12 & 56 \\
无水硫酸镁 & 12 & 87 \\
无水硫酸铜 & 12 & $73^{c}$ \\
\hline
\end{tabular}

脱水剂用量为原料摩尔量的 1.2 倍, 在回流条件下进行反应; ${ }^{b}$ 均为分离产 率; ${ }^{c}$ 芐胺用量增加至取代苯并 $[d][1,3]$ 惡嗪-4-酮的 3 倍.

而本文报道的合成方法同样适用于 3-苠氨基取代喹唑 啉酮化合物的合成. 6-氯-3-苠氨基-2-新戊基-8-甲基喹 唑啉-4(3H)-酮经此方法以苯并噁嗪酮与苠肼在无水硫 酸镁为脱水剂条件下反应, 经过简单后处理, 直接得块 状单晶, 收率达到 $88 \%$. X 光单晶衍射实验对其结构进 行了测定, 得到的分子晶胞结构如图 1 所示, 晶胞内两 分子间有两个氢键相互作用而形成稳定的晶体.

\section{2 生物活性}

对所有目标化合物分别测试了杀虫活性(600 $\mathrm{mg} / \mathrm{L})$ 、抗肿瘤活性 $(5 \mathrm{mg} / \mathrm{L})$ 以及离体 $(200 \mathrm{mg} / \mathrm{L})$ 和活体

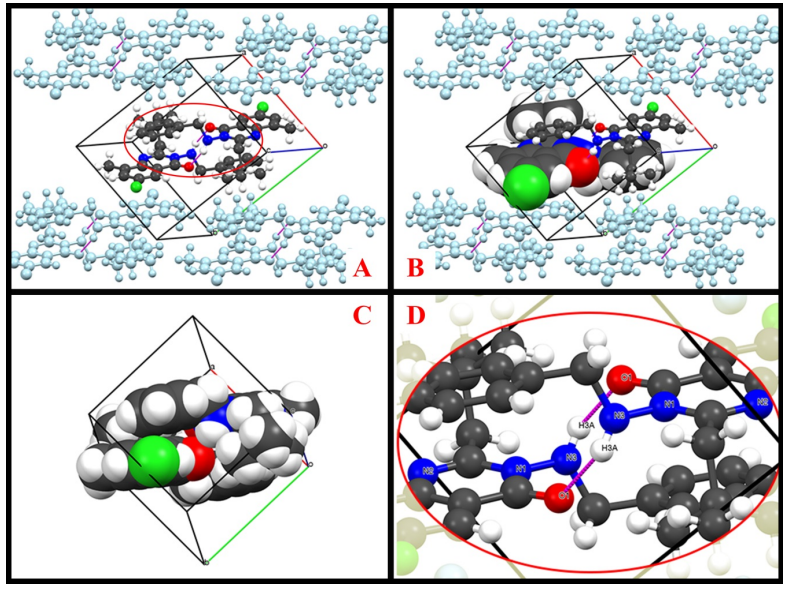

图 1 化合物 6-氯-3-芐氨基-2-新戊基-8-甲基喹唑啉-4(3H)-酮 晶胞及氢键作用中心图

Figure 1 Crystal cell and hydrogen bond of 6-chloro-3- benzylamino-2-neopentyl-8-methylquinazolin-4(3H)-one

A: Crystal unit cell. B: One molecule in the unit cell is shown as molecular surfaces. C: Surfaces of the unit cell. D: Hydrogen bond (in purple) between the two molecules in the unit cell

(200 mg/L)杀菌活性. 其中杀虫及抗肿瘤活性列于表 3, 离体杀菌活性列于表 4, 活体杀菌活性列于表 5 .

表 3 化合物的杀虫和抗癌活性

Table 3 Insecticidal and anti-tumor activities of compounds

\begin{tabular}{|c|c|c|c|c|c|}
\hline \multirow{2}{*}{ 化合物 } & \multicolumn{2}{|c|}{ 对不同试虫致死率 ${ }^{a} / \%$} & \multicolumn{3}{|c|}{ 对不同癌细胞抑制率 ${ }^{b} / \%$} \\
\hline & 瓜蚜 & 小菜蛾 & A-549 & Bel7402 & HCT-8 \\
\hline Q-01 & 41.1 & 33.3 & 9.56 & 5.05 & 5.6 \\
\hline Q-02 & 11.0 & 15.1 & 9.27 & 8.72 & 3.29 \\
\hline Q-03 & 52.2 & 18.2 & 5.22 & 1.53 & 3.51 \\
\hline Q-04 & 47.6 & 25.1 & 10.13 & 1.2 & 12.12 \\
\hline Q-05 & 30.7 & 23.8 & 8.45 & 2.26 & 4.4 \\
\hline Q-06 & 22.5 & 32.7 & 12.57 & 5.75 & 6.99 \\
\hline Q-07 & 65.9 & 24.6 & 7.44 & 2.81 & 7.48 \\
\hline Q-08 & 46.4 & 19.2 & 7.71 & 1.44 & 2.5 \\
\hline Q-09 & 14.2 & 11.3 & 2.96 & 4.69 & 15.51 \\
\hline Q-10 & 31.1 & 26.3 & 4 & 7.11 & 9.51 \\
\hline Q-11 & 14.2 & 21.5 & 18.08 & 15.2 & 0.77 \\
\hline Q-12 & 3.6 & 21.8 & 14.67 & 4.44 & 20.26 \\
\hline Q-13 & 44.4 & 33.5 & -3.58 & 3.74 & 30.62 \\
\hline Q-18 & 37.0 & 47.6 & 13.54 & 0.56 & 11.88 \\
\hline Q-19 & 15.1 & 32.5 & 5.25 & 10.38 & 0.68 \\
\hline Q-20 & 14.1 & 33.3 & 15.07 & 13.93 & 11.42 \\
\hline Q-21 & 14.1 & 14.2 & 11.07 & -1.67 & 3.55 \\
\hline Q-22 & 18.1 & 12.3 & 11.54 & -0.12 & 16.85 \\
\hline Q-23 & 28.4 & 24.1 & 10.86 & -3.76 & 5.79 \\
\hline Q-24 & 50.0 & 29.3 & 9.32 & -2.95 & 8.28 \\
\hline Q-25 & 49.6 & 37.5 & 14.53 & 5.86 & 2.65 \\
\hline Q-26 & 27.2 & 1.5 & 8.13 & 9.98 & 1 \\
\hline Q-27 & 26.2 & 16.2 & 9.51 & -5.44 & 6.76 \\
\hline O-28 & 14.5 & 8.9 & 5.95 & -0.19 & 9.44 \\
\hline Q-29 & 49.8 & 15.2 & 3.78 & 4.77 & 3.24 \\
\hline Q-30 & -2.1 & 22.8 & 12.42 & -5.38 & 2.98 \\
\hline Q-31 & 42.1 & 15.9 & 6.69 & -2.32 & 6.28 \\
\hline
\end{tabular}




\begin{tabular}{|c|c|c|c|c|c|}
\hline \multirow{2}{*}{ 化合物 } & \multicolumn{2}{|c|}{ 对不同试虫致死率 ${ }^{a} / \%$} & \multicolumn{3}{|c|}{ 对不同癌细胞抑制率 ${ }^{b} / \%$} \\
\hline & 瓜蚜 & 小菜蛾 & A-549 & Bel7402 & HCT-8 \\
\hline 吡虫啉 & $100^{c}$ & - & - & - & - \\
\hline 氯虫苯甲酰胺 & - & $100^{d}$ & - & - & - \\
\hline 5-氟尿嘧啶 & - & - & $65.39^{e}$ & $51.82^{e}$ & $59.32^{e}$ \\
\hline
\end{tabular}

表 4 化合物的离体杀菌活性 $\left(200 \mathrm{mg} \cdot \mathrm{L}^{-1}, \%\right)$

Table 4 Fungicidal activities in vitro of compounds at a concentration of $200 \mathrm{mg} \cdot \mathrm{L}^{-1}(\%)$

\begin{tabular}{ccccccccccc}
\hline 化合物 & $\begin{array}{c}\text { 油菜菌 } \\
\text { 核病 }\end{array}$ & $\begin{array}{c}\text { 黄瓜灰 } \\
\text { 霉病 }\end{array}$ & $\begin{array}{c}\text { 水稻纹 } \\
\text { 枯病 }\end{array}$ & $\begin{array}{c}\text { 马铃薯晚 } \\
\text { 疫病 }\end{array}$ & $\begin{array}{c}\text { 黄瓜枯 } \\
\text { 萎病 }\end{array}$ & $\begin{array}{c}\text { 花生褐 } \\
\text { 斑病 }\end{array}$ & $\begin{array}{c}\text { 苹果轮 } \\
\text { 纹病 }\end{array}$ & $\begin{array}{c}\text { 番茄早 } \\
\text { 疫病 }\end{array}$ & $\begin{array}{c}\text { 小麦赤 } \\
\text { 霉病 }\end{array}$ & $\begin{array}{c}\text { 辣椒疫 } \\
\text { 霉病 }\end{array}$ \\
\hline Q-01 & 30.0 & 34.8 & 51.7 & 33.3 & 38.9 & 37.5 & 36.6 & 27.8 & 23.1 & 25.0 \\
Q-02 & 23.3 & 21.7 & 24.1 & 16.7 & 16.7 & 12.5 & 26.8 & 16.7 & 34.6 & 29.2 \\
Q-03 & 13.3 & 21.7 & 55.2 & 16.7 & 16.7 & 18.8 & 43.9 & 22.2 & 34.6 & 25.0 \\
Q-05 & 46.7 & 21.7 & 44.8 & 25.0 & 22.2 & 18.8 & 12.2 & 16.7 & 38.5 & 33.3 \\
Q-06 & 46.7 & 21.7 & 41.4 & 16.7 & 27.8 & 25.0 & 12.2 & 22.2 & 30.8 & 16.7 \\
Q-07 & 23.3 & 26.1 & 65.5 & 25.0 & 33.3 & 25.0 & 31.7 & 16.7 & 34.6 & 12.5 \\
Q-09 & 26.7 & 26.1 & 37.9 & 25.0 & 33.3 & 25.0 & 24.4 & 16.7 & 30.8 & 20.8 \\
Q-10 & 30.0 & 30.4 & 41.4 & 12.5 & 33.3 & 25.0 & 48.8 & 16.7 & 46.2 & 20.8 \\
Q-13 & 30.0 & 21.7 & 37.9 & 25.0 & 11.1 & 25.0 & 12.2 & 22.2 & 26.9 & 33.3 \\
Q-18 & 30.0 & 21.7 & 41.4 & 12.5 & 11.1 & 12.5 & 12.2 & 22.2 & 46.2 & 12.5 \\
Q-19 & 43.3 & 30.4 & 44.8 & 12.5 & 27.8 & 18.8 & 36.6 & 11.1 & 38.5 & 16.7 \\
Q-20 & 30.0 & 13.0 & 41.4 & 12.5 & 16.7 & 12.5 & 48.8 & 11.1 & 34.6 & 20.8 \\
Q-21 & 43.3 & 30.4 & 41.4 & 12.5 & 27.8 & 18.8 & 41.5 & 16.7 & 19.2 & 20.8 \\
Q-22 & 13.3 & 21.7 & 34.5 & 12.5 & 16.7 & 25.0 & 31.7 & 22.2 & 34.6 & 16.7 \\
Q-23 & 13.3 & 17.4 & 10.3 & 29.2 & 22.2 & 12.5 & 43.9 & 11.1 & 19.2 & 16.7 \\
Q-24 & 26.7 & 30.4 & 44.8 & 25.0 & 33.3 & 43.8 & 12.2 & 44.4 & 38.5 & 29.2 \\
Q-25 & 13.3 & 17.4 & 27.6 & 12.5 & 22.2 & 25.0 & 34.1 & 22.2 & 26.9 & 20.8 \\
Q-27 & 30.0 & 30.4 & 27.6 & 25.0 & 38.9 & 37.5 & 46.3 & 33.3 & 42.3 & 12.5 \\
Q-29 & 13.3 & 26.1 & 55.2 & 25.0 & 11.1 & 12.5 & 26.8 & 11.1 & 30.8 & 20.8 \\
Q-30 & 46.7 & 30.4 & 41.4 & 12.5 & 11.1 & 12.5 & 43.9 & 11.1 & 34.6 & 20.8 \\
Q-31 & 36.7 & 21.7 & 44.8 & 16.7 & 16.7 & 18.8 & 14.6 & 22.2 & 38.5 & 25.0 \\
\hline
\end{tabular}

表 5 化合物的活体杀菌活性 $\left(200 \mathrm{mg} \cdot \mathrm{L}^{-1}, \%\right)$

Table 5 Fungicidal activities in vivo of compounds at a concentration of $200 \mathrm{mg} \cdot \mathrm{L}^{-1}(\%)$

\begin{tabular}{ccc||ccc}
\hline 化合物 & 小麦白粉病 & 辣椒疫霉病 & 化合物 & 小麦白粉病 & 辣椒疫霉病 \\
\hline Q-01 & 30.0 & - & $\mathbf{Q - 2 1}$ & 60.0 & 50.0 \\
Q-02 & 50.0 & - & $\mathbf{Q - 2 2}$ & 40.0 & 58.3 \\
Q-03 & 50.0 & 58.3 & $\mathbf{Q - 2 3}$ & 40.0 & 41.7 \\
Q-05 & 50.0 & - & $\mathbf{Q - 2 4}$ & 99.0 & 37.5 \\
Q-06 & 75.0 & 16.7 & $\mathbf{Q - 2 5}$ & 60.0 & 58.3 \\
Q-07 & 60.0 & 45.8 & $\mathbf{Q - 2 7}$ & 90.0 & 50.0 \\
Q-09 & 70.0 & 35.0 & $\mathbf{Q - 2 9}$ & 60.0 & 50.0 \\
Q-10 & 50.0 & 29.2 & $\mathbf{Q - 3 1}$ & 70.0 & 33.3 \\
Q-13 & 30.0 & - & 苯醚甲环唑 & 80.0 & - \\
Q-18 & 60.0 & 37.5 & 烯酰吗啉 & $99.0^{a}$ & - \\
Q-19 & - & 58.3 & & & $100^{b}$ \\
Q-20 & 55.0 & 50.0 & & & \\
\hline
\end{tabular}

苯醚甲环唑浓度为 $100 \mathrm{mg} \cdot \mathrm{L}^{-1} ;^{b}$ 烯酰吗啉浓度为 $12.5 \mathrm{mg} \cdot \mathrm{L}^{-1}$.

生物活性测试结果表明, 该系列化合物不具有抗癌 和杀虫活性, 但是大部分化合物在 $200 \mathrm{mg} \cdot \mathrm{L}^{-1}$ 浓度下对 小麦白粉病具有较好的活体防效, 其中化合物 Q-24, Q-27 和 Q-31, 分别达到了 $99 \%, 90 \%$ 和 $80 \%$.
从杀菌活性初篮结果分析，化合物 8-位的甲基取代 $\left(\mathrm{R}^{1}\right)$ 能稍微增加化合物防治小麦白粉病的活性，如化合 物 Q-23 与化合物 Q-27 相比较, 化合物 Q-23 的活性仅 为 40\%, 而 $\mathrm{Q}-27$ 为 50\%. $\mathrm{R}^{2}$ 位置的取代基为正丁基时活 
性最高, 如化合物 Q-20 与 $\mathbf{Q - 2 4}$ 相比, $\mathrm{R}^{2}$ 基团仅由正丁 基变为正戍基时，化合物活性从 99\%降低至 55\%; 将 $\mathrm{R}^{2}$ 基团变换为环丙基或环己基取代时化合物活性亦有不 同程度的降低. 在 $\mathrm{R}^{3}$ 基团的间位引入氟原子能提高化 合物活性，而邻位或对位氟原子取代对活性没有贡献. 如化合物 Q-29, Q-30 和 Q-31, 其中化合物活性最高的 是在苯环上间位引入氟原子的化合物 Q-31, 达到了 $90 \%$, 在苯环邻和对位引入氟原子时, $\mathbf{Q - 3 0}$ 与 $\mathbf{Q - 2 9}$ 活性 分别仅为 $70 \%$ 与 $60 \%$.

\section{2 结论}

本文优化合成喹唑啉-4(3H)-酮类化合物的反应中, 发现加入一定量常用干燥剂(无水硫酸钠、无水硫酸镁、 无水硫酸铜和无水氯化钙等)能够提高目标产物的生成, 相关方法合成喹唑啉酮类化合物未见文献报道. 采用该 方法以取代静红酸䣶为起始原料, 合成了 2,3-二取代喹 唑啉-4(3H)-酮化合物 31 个, 仅有两个已报道化合物(但 均未报道生物活性数据), 其余均为新化合物. 化合物 均通过了核磁共振氢谱、核磁共振碳谱和高分辨质谱等 方法的结构表征.

获得了 6-氯-3-芐氨基-2-新戊基-8-甲基喹唑啉$4(3 H)$-酮的晶体，通过 X 衍射对该化合物的晶体结构进 行了检测, 解析的单晶结构数据对喹唑啉酮类化合物构 效关系研究及活性化合物设计具有重要的指导意义.

对新化合物的杀虫、抗癌以及杀菌等生物活性进行 了测试, 发现大部分化合物在 $200 \mathrm{mg} \cdot \mathrm{L}^{-1}$ 浓度下对小麦 白粉病具有一定的活体防效, 其中化合物 Q-24, Q-27 和 Q-31 的防效分别达到了 $99 \%, 90 \%$ 和 $80 \%$. 通过对化合 物杀菌活性的构效关系初步研究发现, 在化合物 $\mathrm{R}^{3}$ 基 团苯基间位的氟原子对化合物杀菌活性有重要影响.

\section{3 实验部分}

\section{1 仪器与试剂}

熔点测定仪: Stuart SMP-3 熔点测定仪(未校正); 核 磁共振仪: Bruker Avance DPX300, $\mathrm{CDCl}_{3}$ 或 DMSO-d $\mathrm{d}_{6}$ 为溶剂, TMS 为内标; LC-MS: Agilent G1956B (EI 源); HRMS:Agilent G1969A (EI 源). 所有试剂均为市售分析 纯，从 Alfa-Asear， J\&K 或北京偶合科技有限公司购买， 无水三乙胺和无水四氢呋喃都是通过标准方法 ${ }^{[19]}$ 进行 无水处理后使用, 其它试剂及溶剂使用前均未经进一步 纯化.

\section{2 化合物的合成}

\subsection{1 苯并噁嗪酮中间体 $\mathbf{M}$ 的合成}

2-丁基-4H-苯并 $[d][1,3]$ 哑溙-4-酮的合成: $100 \mathrm{~mL}$ 三 口圆底烧瓶中, 依次加入 $5.0 \mathrm{~g}(30 \mathrm{mmol})$ 静红酸䣲 $(1 \mathrm{H}-$
苯并 $[d][1,3]$ 噁嗪-2,4-二酮), $50 \mathrm{~mL}$ 无水四氢呋喃和 $9.3 \mathrm{~g}$ $(90 \mathrm{mmol})$ 无水三乙胺. 将反应液搅拌条件下冰浴降温 至 $0{ }^{\circ} \mathrm{C}$, 在 $30 \mathrm{~min}$ 内用注射葲将 $4.4 \mathrm{~g} \mathrm{(36} \mathrm{mmol)}$ 正戊酰 氯缓慢注入反应瓶中, 然后室温摚拌反应 $12 \mathrm{~h}$. 反应完 毕后, 旋蒸除去大部分四氢呋喃和三乙胺, 残余油状物 用 $50 \mathrm{~mL}$ 乙酸乙酯溶解, 水和饱和碳酸氢钠溶液分别洗 涤两次后 $(50 \mathrm{~mL} \times 2)$, 无水硫酸钠干燥, 蒸干溶剂得白 色固体 5.7 g, 产率 91\%. m.p. 75.3 75.9 ${ }^{\circ} \mathrm{C} ;{ }^{1} \mathrm{H}$ NMR $\left(300 \mathrm{MHz}, \mathrm{CDCl}_{3}\right) \delta: 8.18(\mathrm{dd}, J=7.9,1.9 \mathrm{~Hz}, 1 \mathrm{H}, \mathrm{Ar}-\mathrm{H})$, $7.82 \sim 7.76(\mathrm{~m}, 1 \mathrm{H}, \mathrm{Ar}-\mathrm{H}), 7.57 \sim 7.46(\mathrm{~m}, 2 \mathrm{H}, \mathrm{Ar}-\mathrm{H}), 2.69$ $\left(\mathrm{t}, J=7.9 \mathrm{~Hz}, 2 \mathrm{H}, \mathrm{CH}_{2}\right), 1.87 \sim 1.76\left(\mathrm{~m}, 2 \mathrm{H}, \mathrm{CH}_{2}\right), 1.50 \sim$ $1.42\left(\mathrm{~m}, 2 \mathrm{H}, \mathrm{CH}_{2}\right), 0.97$ (t, $\left.J=7.4 \mathrm{~Hz}, \mathrm{CH}_{3}\right)$; EI-MS $m / z$ (\%): $203\left(\mathrm{M}^{+}, 100\right)$.

中间体 2-戊基-4H-苯并 $[d][1,3]$ 哑嗪-4-酮, 2-丁基-8甲基- $4 \mathrm{H}$-苯并 $[d][1,3]$ 啞嗪-4-酮及 2 -环丙基- $4 H$-苯并 $[d][1,3]$ 噁嗪-4-酮合成步骤和后处理方法均与 2-丁基$4 H$-苯并 $[d][1,3]$ 惡嗪-4-酮相同, 相关数据如下:

2-戊基- $4 H$-苯并 $[d][1,3]$ 噁嗪-4-酮：白色固体，产率 $90 \%$. m.p. $76.7 \sim 78.3{ }^{\circ} \mathrm{C} ;{ }^{1} \mathrm{H}$ NMR $\left(300 \mathrm{MHz}, \mathrm{CDCl}_{3}\right) \delta$ : $8.18(\mathrm{dd}, J=7.9,1.9 \mathrm{~Hz}, 1 \mathrm{H}, \mathrm{Ar}-\mathrm{H}), 7.82 \sim 7.76(\mathrm{~m}, 1 \mathrm{H}$, Ar-H), $7.58 \sim 7.46(\mathrm{~m}, 2 \mathrm{H}, \mathrm{Ar}-\mathrm{H}), 2.69(\mathrm{t}, J=7.5 \mathrm{~Hz}, 2 \mathrm{H}$, $\left.\mathrm{CH}_{2}\right), 1.89 \sim 1.85\left(\mathrm{~m}, 2 \mathrm{H}, \mathrm{CH}_{2}\right), 1.44 \sim 1.34\left(\mathrm{~m}, 4 \mathrm{H}, \mathrm{CH}_{2}\right)$, $0.95 \sim 0.89$ (m, 3H, $\left.\mathrm{CH}_{3}\right)$; EI-MS $m / z(\%): 217\left(\mathrm{M}^{+}, 100\right)$.

2-丁基-8-甲基- $4 H$-苯并 $[d][1,3]$ 噁嗪-4-酮：白色固 体, 产率 95\%. m.p. 97.3 99.1 ${ }^{\circ} \mathrm{C} ;{ }^{1} \mathrm{H}$ NMR $(300 \mathrm{MHz}$, $\left.\mathrm{CDCl}_{3}\right) \delta: 8.03(\mathrm{dd}, J=7.9,0.9 \mathrm{~Hz}, 1 \mathrm{H}, \mathrm{Ar}-\mathrm{H}), 7.63(\mathrm{dd}$, $J=7.9,0.9 \mathrm{~Hz}, 1 \mathrm{H}, \mathrm{Ar}-\mathrm{H}), 7.40 \sim 7.33$ (m, 2H, Ar-H), 2.69 (t, $\left.J=7.8 \mathrm{~Hz}, 2 \mathrm{H}, \mathrm{CH}_{2}\right), 2.54$ (s, $\left.3 \mathrm{H}, \mathrm{Ar}-\mathrm{CH}_{3}\right), 1.87 \sim 1.77$ $\left(\mathrm{m}, 2 \mathrm{H}, \mathrm{CH}_{2}\right), 1.53 \sim 1.40\left(\mathrm{~m}, 2 \mathrm{H}, \mathrm{CH}_{2}\right), 0.97$ (t, $J=7.3$ $\left.\mathrm{Hz}, 3 \mathrm{H}, \mathrm{CH}_{3}\right)$; EI-MS $m / z(\%): 217\left(\mathrm{M}^{+}, 100\right)$.

2-环丙基-4H-苯并 $[d][1,3]$ 噁嗪-4-酮：淡黄色粉末， 产率 81\%. m.p. 102.1 104.0 ${ }^{\circ} \mathrm{C}$; ${ }^{1} \mathrm{H}$ NMR $(300 \mathrm{MHz}$, $\left.\mathrm{CDCl}_{3}\right) \delta: 8.17 \sim 8.13(\mathrm{~m}, 1 \mathrm{H}, \mathrm{Ar}-\mathrm{H}), 7.77 \sim 7.73(\mathrm{~m}, 1 \mathrm{H}$, Ar-H), $7.52 \sim 7.41(\mathrm{~m}, 2 \mathrm{H}, \mathrm{Ar}-\mathrm{H}), 2.00 \sim 1.93(\mathrm{~m}, 1 \mathrm{H}$, $\mathrm{CH}), 1.33 \sim 1.26\left(\mathrm{~m}, 2 \mathrm{H}, \mathrm{CH}_{2}\right), 1.14 \sim 1.11\left(\mathrm{~m}, 2 \mathrm{H}, \mathrm{CH}_{2}\right)$; EI-MS $m / z(\%): 187\left(\mathrm{M}^{+}, 100\right)$.

\subsection{2 目标化合物 $\mathbf{Q}$ 的合成}

以 2-丁基-3-(吡定-2-基甲基)喹唑啉-4(3H)-酮(Q-01) 的合成为例:

$25 \mathrm{~mL}$ 圆底烧瓶中, 依次加入 $0.20 \mathrm{~g}(1 \mathrm{mmol})$ 中间 体 2-丁基-4H-苯并 $[d][1,3]$ 噁嗪-4-酮, $0.072 \mathrm{~g}(0.6 \mathrm{mmol})$ 无水硫酸镁和 $10 \mathrm{~mL}$ 无水四氢呋喃. 搅拌下加入 $0.13 \mathrm{~g}$ (1.2 mmol) 2-氨甲基吡啶, 加热搅拌回流反应 $8 \mathrm{~h}$. TLC 监测反应完毕后，用装有 $2 \mathrm{~cm}$ 硅胶层的漏斗过滤，四氢 呋喃洗涤硅胶层, 收集滤液加入石油醚结晶, 过滤得 2- 
丁基-3-(吡啶-2-基甲基)喹唑啉-4(3H)-酮(Q-01): 白色粉 末 $0.25 \mathrm{~g}$, 收率 $87 \%$. m.p. $112.2 \sim 112.7{ }^{\circ} \mathrm{C} ;{ }^{1} \mathrm{H}$ NMR $\left(300 \mathrm{MHz}, \mathrm{CDCl}_{3}\right) \delta: 8.57$ (br, $\left.2 \mathrm{H}, \mathrm{ArH}\right), 8.33 \sim 8.24(\mathrm{~m}$, $1 \mathrm{H}, \mathrm{ArH}), 7.83 \sim 7.70(\mathrm{~m}, 1 \mathrm{H}, \mathrm{ArH}), 7.66$ (dd, $J=8.2,1.2$ $\mathrm{Hz}, 1 \mathrm{H}, \mathrm{ArH}), 7.58 \sim 7.50(\mathrm{~m}, 1 \mathrm{H}, \mathrm{ArH}), 7.50 \sim 7.41(\mathrm{~m}$, $1 \mathrm{H}, \mathrm{ArH}), 7.32 \sim 7.14(\mathrm{~m}, 1 \mathrm{H}, \mathrm{ArH}), 5.41\left(\mathrm{~s}, 2 \mathrm{H}, \mathrm{CH}_{2}\right)$, $2.83 \sim 2.67\left(\mathrm{~m}, 2 \mathrm{H}, \mathrm{CH}_{2}\right), 1.86 \sim 1.71\left(\mathrm{~m}, 2 \mathrm{H}, \mathrm{CH}_{2}\right)$, $1.50 \sim 1.34\left(\mathrm{~m}, 2 \mathrm{H}, \mathrm{CH}_{2}\right), 0.93\left(\mathrm{t}, J=7.3 \mathrm{~Hz}, 3 \mathrm{H}, \mathrm{CH}_{3}\right)$; ${ }^{13} \mathrm{C}$ NMR $\left(75 \mathrm{MHz}, \mathrm{CDCl}_{3}\right) \delta: 162.30,156.41,148.92$, $148.11,147.10,134.24,132.00,126.90,126.72,126.38$, $123.58,120.01,44.04,34.71,28.96,22.18,13.59$. HRMS (EI) calcd for $\mathrm{C}_{18} \mathrm{H}_{20} \mathrm{~N}_{3} \mathrm{O}[\mathrm{M}+\mathrm{H}]^{+}$294.1606, found 294.1608.

目标化合物 $\mathbf{Q - 0 2} \sim \mathbf{Q - 3 1}$ 反应步骤和后处理方法与 Q-01 相似, 理化数据如下:

2-丁基-3-(4-氯苄基)喹唑啉-4(3H)-酮(Q-02): 白色 粉末, 收率 90\%. m.p. 96.3 97.6 ${ }^{\circ} \mathrm{C} ;{ }^{1} \mathrm{H}$ NMR (300 $\left.\mathrm{MHz}, \mathrm{CDCl}_{3}\right) \delta: 8.36 \sim 8.24(\mathrm{~m}, 1 \mathrm{H}, \mathrm{ArH}), 7.82 \sim 7.62(\mathrm{~m}$, $2 \mathrm{H}, \mathrm{ArH}), 7.51 \sim 7.42(\mathrm{~m}, 1 \mathrm{H}, \mathrm{ArH}), 7.36 \sim 7.27(\mathrm{~m}, 2 \mathrm{H}$, $\operatorname{ArH}), 7.18 \sim 7.09(\mathrm{~m}, 2 \mathrm{H}, \mathrm{ArH}), 5.37\left(\mathrm{~s}, 2 \mathrm{H}, \mathrm{CH}_{2}\right), 2.85 \sim$ $2.60\left(\mathrm{~m}, 2 \mathrm{H}, \mathrm{CH}_{2}\right), 1.85 \sim 1.68\left(\mathrm{~m}, 2 \mathrm{H}, \mathrm{CH}_{2}\right), 1.46 \sim 1.33$ (m, $2 \mathrm{H}, \mathrm{CH}_{2}$ ), 0.92 (t, $\left.J=7.3 \mathrm{~Hz}, 3 \mathrm{H}, \mathrm{CH}_{3}\right) ;{ }^{13} \mathrm{C}$ NMR (75 $\left.\mathrm{MHz}, \mathrm{CDCl}_{3}\right) \delta: 162.47,157.09,147.22,134.78,134.43$, $133.53,129.08,127.85,127.03,126.96,126.56,120.23$, 45.90, 34.89, 29.31, 22.43, 13.76. HRMS (EI) calcd for $\mathrm{C}_{19} \mathrm{H}_{20} \mathrm{ClN}_{2} \mathrm{O}[\mathrm{M}+\mathrm{H}]^{+}$327.1264, found 327.1262.

3-(3-溴苄基)-2-丁基喹唑啉-4(3H)-酮(Q-03): 白色 晶体, 收率 91\%. m.p. 99.5 100.3 ${ }^{\circ} \mathrm{C} ;{ }^{1} \mathrm{H}$ NMR (300 $\left.\mathrm{MHz}, \mathrm{CDCl}_{3}\right) \delta: 8.37 \sim 8.20(\mathrm{~m}, 1 \mathrm{H}, \mathrm{ArH}), 7.77 \sim 7.64(\mathrm{~m}$, $2 \mathrm{H}, \mathrm{ArH}), 7.47 \sim 7.37(\mathrm{~m}, 2 \mathrm{H}, \mathrm{ArH}), 7.33(\mathrm{~d}, J=1.4 \mathrm{~Hz}$, $1 \mathrm{H}, \mathrm{ArH}), 7.21 \sim 7.15(\mathrm{~m}, 1 \mathrm{H}, \mathrm{ArH}), 7.11 \sim 7.06(\mathrm{~m}, 1 \mathrm{H}$, $\mathrm{ArH}), 5.37(\mathrm{~s}, 2 \mathrm{H}), 2.78 \sim 2.65\left(\mathrm{~m}, 2 \mathrm{H}, \mathrm{CH}_{2}\right), 1.82 \sim 1.69$ $\left(\mathrm{m}, 2 \mathrm{H}, \mathrm{CH}_{2}\right), 1.45 \sim 1.32\left(\mathrm{~m}, 2 \mathrm{H}, \mathrm{CH}_{2}\right), 0.91(\mathrm{t}, J=7.3$ $\mathrm{Hz}, 3 \mathrm{H}, \mathrm{CH}_{3}$ ). HRMS (EI) calcd for $\mathrm{C}_{19} \mathrm{H}_{20} \mathrm{BrN}_{2} \mathrm{O}[\mathrm{M}+\mathrm{H}]^{+}$ 371.0759 , found 371.0758 .

3-(4-溴苄基)-2-丁基喹唑啉-4(3H)-酮(Q-04): 白色 粉末, 收率 $83 \%$. m.p. 105.3 105.8 ${ }^{\circ} \mathrm{C} ;{ }^{1} \mathrm{H}$ NMR (300 $\left.\mathrm{MHz}, \mathrm{CDCl}_{3}\right) \delta: 8.36 \sim 8.23(\mathrm{~m}, 1 \mathrm{H}, \mathrm{ArH}), 7.82 \sim 7.62(\mathrm{~m}$, $2 \mathrm{H}, \mathrm{ArH}), 7.52 \sim 7.39(\mathrm{~m}, 3 \mathrm{H}, \mathrm{ArH}), 7.15 \sim 6.98(\mathrm{~m}, 2 \mathrm{H}$, $\mathrm{ArH}), 5.34\left(\mathrm{~s}, 2 \mathrm{H}, \mathrm{CH}_{2}\right), 2.82 \sim 2.62\left(\mathrm{~m}, 2 \mathrm{H}, \mathrm{CH}_{2}\right), 1.86 \sim$ $1.66\left(\mathrm{~m}, 2 \mathrm{H}, \mathrm{CH}_{2}\right), 1.48 \sim 1.33\left(\mathrm{~m}, 2 \mathrm{H}, \mathrm{CH}_{2}\right), 0.92(\mathrm{t}, J=$ $\left.7.3 \mathrm{~Hz}, 3 \mathrm{H}, \mathrm{CH}_{3}\right) ;{ }^{13} \mathrm{C}$ NMR $\left(75 \mathrm{MHz}, \mathrm{CDCl}_{3}\right) \delta: 162.41$, $156.96,147.22,135.29,134.35,131.96,128.12,126.95$, $126.48,121.48,120.18,77.42,77.00,76.58,45.88,34.85$, 29.21, 22.37, 13.73. HRMS (EI) calcd for $\mathrm{C}_{19} \mathrm{H}_{20} \mathrm{BrN}_{2} \mathrm{O}$
$[\mathrm{M}+\mathrm{H}]^{+}$371.0759, found 371.0755.

2-丁基-3-(4-甲基芐基)喹唑啉-4(3H)-酮(Q-05): 白 色粉末, 收率 88\%. m.p. 113.1 114.1 ${ }^{\circ} \mathrm{C} ;{ }^{1} \mathrm{H}$ NMR (300 $\left.\mathrm{MHz}, \mathrm{CDCl}_{3}\right) \delta: 8.34 \sim 8.27(\mathrm{~m}, 1 \mathrm{H}, \mathrm{ArH}), 7.76 \sim 7.63(\mathrm{~m}$, $2 \mathrm{H}, \mathrm{ArH}), 7.48 \sim 7.40(\mathrm{~m}, 1 \mathrm{H}, \mathrm{ArH}), 7.16 \sim 7.04(\mathrm{~m}, 4 \mathrm{H}$, $\mathrm{ArH}), 5.37\left(\mathrm{~s}, 2 \mathrm{H}, \mathrm{CH}_{2}\right), 2.80 \sim 2.70\left(\mathrm{~m}, 2 \mathrm{H}, \mathrm{CH}_{2}\right), 2.30(\mathrm{~s}$, $\left.3 \mathrm{H}, \mathrm{CH}_{3}\right), 1.81 \sim 1.68(\mathrm{~m}, 2 \mathrm{H}), 1.46 \sim 1.32\left(\mathrm{~m}, 2 \mathrm{H}, \mathrm{CH}_{2}\right)$, 0.91 (t, $J=7.3 \mathrm{~Hz}, 3 \mathrm{H}, \mathrm{CH}_{3}$ ). HRMS (EI) calcd for $\mathrm{C}_{20} \mathrm{H}_{23} \mathrm{~N}_{2} \mathrm{O}[\mathrm{M}+\mathrm{H}]^{+}$307.1810, found 307.1811.

2-丁基-3-(2-甲基芐基)喹唑啉-4(3H)-酮(Q-06): 白 色粉末, 收率 $81 \%$. m.p. $94.8 \sim 95.8{ }^{\circ} \mathrm{C} ;{ }^{1} \mathrm{H}$ NMR (300 $\left.\mathrm{MHz}, \mathrm{CDCl}_{3}\right) \delta: 8.34 \sim 8.26(\mathrm{~m}, 1 \mathrm{H}, \mathrm{ArH}), 7.80 \sim 7.65(\mathrm{~m}$, $2 \mathrm{H}, \mathrm{ArH}), 7.50 \sim 7.42(\mathrm{~m}, 1 \mathrm{H}, \mathrm{ArH}), 7.24 \sim 7.12(\mathrm{~m}, 2 \mathrm{H}$, $\mathrm{ArH}), 7.12 \sim 7.02(\mathrm{~m}, 1 \mathrm{H}, \mathrm{ArH}), 6.72 \sim 6.58(\mathrm{~m}, 1 \mathrm{H}, \mathrm{ArH})$, $5.35\left(\mathrm{~s}, 2 \mathrm{H}, \mathrm{CH}_{2}\right), 2.76 \sim 2.56\left(\mathrm{~m}, 2 \mathrm{H}, \mathrm{CH}_{2}\right), 2.43(\mathrm{~s}, 3 \mathrm{H}$, $\left.\mathrm{CH}_{3}\right), 1.86 \sim 1.69\left(\mathrm{~m}, 2 \mathrm{H}, \mathrm{CH}_{2}\right), 1.44 \sim 1.28\left(\mathrm{~m}, 2 \mathrm{H}, \mathrm{CH}_{2}\right)$, $0.90\left(\mathrm{t}, J=7.3 \mathrm{~Hz}, 3 \mathrm{H}, \mathrm{CH}_{3}\right) ;{ }^{13} \mathrm{C} \mathrm{NMR}\left(75 \mathrm{MHz}, \mathrm{CDCl}_{3}\right)$ $\delta: 161.52,160.73,145.96,138.51,134.80,134.54,131.91$, $130.09,128.82,127.86,126.55,126.15,124.45,121.15$, 45.56, 42.43, 31.41, 25.90, 25.47. HRMS (EI) calcd for $\mathrm{C}_{20} \mathrm{H}_{23} \mathrm{~N}_{2} \mathrm{O}[\mathrm{M}+\mathrm{H}]^{+}$307.1810, found 307.1813.

2-丁基-3-(3-甲氧基芐基)喹唑啉-4(3H)-酮(Q-07): 白色粉末, 收率 83\%. m.p. 74.7 75.8 ${ }^{\circ} \mathrm{C} ;{ }^{1} \mathrm{H}$ NMR (300 $\left.\mathrm{MHz}, \mathrm{CDCl}_{3}\right) \delta: 8.30(\mathrm{dd}, J=8.0,1.5 \mathrm{~Hz}, 1 \mathrm{H}, \mathrm{ArH})$, $7.76 \sim 7.64(\mathrm{~m}, 2 \mathrm{H}, \operatorname{ArH}), 7.48 \sim 7.40(\mathrm{~m}, 1 \mathrm{H}, \operatorname{ArH})$, $7.27 \sim 7.19(\mathrm{~m}, 1 \mathrm{H}, \mathrm{ArH}), 6.83 \sim 6.70(\mathrm{~m}, 3 \mathrm{H}, \mathrm{ArH}), 5.38$ (s, $\left.2 \mathrm{H}, \mathrm{CH}_{2}\right), 3.75\left(\mathrm{~s}, 3 \mathrm{H}, \mathrm{CH}_{3}\right), 2.81 \sim 2.68\left(\mathrm{~m}, 2 \mathrm{H}, \mathrm{CH}_{2}\right)$, $1.85 \sim 1.66\left(\mathrm{~m}, 2 \mathrm{H}, \mathrm{CH}_{2}\right), 1.47 \sim 1.32\left(\mathrm{~m}, 2 \mathrm{H}, \mathrm{CH}_{2}\right), 0.91$ (t, $J=7.3 \mathrm{~Hz}, 3 \mathrm{H}, \mathrm{CH}_{3}$ ). HRMS (EI) calcd for $\mathrm{C}_{20} \mathrm{H}_{23} \mathrm{~N}_{2} \mathrm{O}_{2}$ $[\mathrm{M}+\mathrm{H}]^{+}$23.1760, found 323.1759.

2-丁基-3-(4-甲氧基芐基)喹唑啉-4(3H)-酮 (Q-08): 白色固体, 收率 82\%. m.p. 102.4 105.1 ${ }^{\circ} \mathrm{C} ;{ }^{1} \mathrm{H}$ NMR $\left(300 \mathrm{MHz}, \mathrm{CDCl}_{3}\right) \delta: 8.44 \sim 8.24(\mathrm{~m}, 1 \mathrm{H}, \mathrm{ArH}), 7.76 \sim$ $7.62(\mathrm{~m}, 2 \mathrm{H}, \mathrm{ArH}), 7.47 \sim 7.40(\mathrm{~m}, 1 \mathrm{H}, \mathrm{ArH}), 7.19 \sim 7.10$ (m, $2 \mathrm{H}, \operatorname{ArH}), 6.87 \sim 6.80(\mathrm{~m}, 2 \mathrm{H}, \operatorname{ArH}), 5.34(\mathrm{~s}, 2 \mathrm{H}$, $\left.\mathrm{CH}_{2}\right), 3.75\left(\mathrm{~s}, 3 \mathrm{H}, \mathrm{CH}_{3}\right), 2.86 \sim 2.71\left(\mathrm{~m}, 2 \mathrm{H}, \mathrm{CH}_{2}\right), 1.89 \sim$ $1.66\left(\mathrm{~m}, 2 \mathrm{H}, \mathrm{CH}_{2}\right), 1.52 \sim 1.31\left(\mathrm{~m}, 2 \mathrm{H}, \mathrm{CH}_{2}\right), 0.92(\mathrm{t}, J=$ $7.3 \mathrm{~Hz}, 3 \mathrm{H}, \mathrm{CH}_{3}$ ). HRMS (EI) calcd for $\mathrm{C}_{20} \mathrm{H}_{23} \mathrm{~N}_{2} \mathrm{O}_{2}[\mathrm{M}+$ $\mathrm{H}]^{+} 323.1760$, found 323.1762 .

3-苄基-2-丁基喹唑啉-4(3H)-酮(Q-09): 白色固体, 收率 79\%. m.p. 81.4 82.2 ${ }^{\circ} \mathrm{C} ;{ }^{1} \mathrm{H}$ NMR $(300 \mathrm{MHz}$, $\left.\mathrm{CDCl}_{3}\right) \delta: 8.44 \sim 8.19(\mathrm{~m}, 1 \mathrm{H}, \mathrm{ArH}), 7.79 \sim 7.64(\mathrm{~m}, 2 \mathrm{H}$, $\mathrm{ArH}), 7.50 \sim 7.41(\mathrm{~m}, 1 \mathrm{H}, \mathrm{ArH}), 7.37 \sim 7.25(\mathrm{~m}, 3 \mathrm{H}, \mathrm{ArH})$, $7.22 \sim 7.12(\mathrm{~m}, 2 \mathrm{H}, \mathrm{ArH}), 5.42\left(\mathrm{~s}, 2 \mathrm{H}, \mathrm{CH}_{2}\right), 2.84 \sim 2.64$ (m, $\left.2 \mathrm{H}, \mathrm{CH}_{2}\right), 1.84 \sim 1.67\left(\mathrm{~m}, 2 \mathrm{H}, \mathrm{CH}_{2}\right), 1.48 \sim 1.30(\mathrm{~m}$, 
$2 \mathrm{H}, \mathrm{CH}_{2}$ ), 0.91 (t, $J=7.3 \mathrm{~Hz}, 3 \mathrm{H}, \mathrm{CH}_{3}$ ). HRMS (EI) calcd for $\mathrm{C}_{19} \mathrm{H}_{21} \mathrm{~N}_{2} \mathrm{O}[\mathrm{M}+\mathrm{H}]^{+}$293.1654, found 393.1653.

2-丁基-3-(4-(叔丁基)芐基)喹唑啉-4(3H)-酮(Q-10): 白色粉末, 收率 $89 \%$. m.p. 87.7 88.5 ${ }^{\circ} \mathrm{C} ;{ }^{1} \mathrm{H}$ NMR (300 $\left.\mathrm{MHz}, \mathrm{CDCl}_{3}\right) \delta: 8.44 \sim 8.13(\mathrm{~m}, 1 \mathrm{H}, \mathrm{ArH}), 7.82 \sim 7.62(\mathrm{~m}$, $2 \mathrm{H}, \mathrm{ArH}), 7.50 \sim 7.40(\mathrm{~m}, 1 \mathrm{H}, \mathrm{ArH}), 7.39 \sim 7.29(\mathrm{~m}, 2 \mathrm{H}$, $\mathrm{ArH}), 7.17 \sim 7.06(\mathrm{~m}, 2 \mathrm{H}, \mathrm{ArH}), 5.38\left(\mathrm{~s}, 2 \mathrm{H}, \mathrm{CH}_{2}\right), 2.94 \sim$ $2.62\left(\mathrm{~m}, 2 \mathrm{H}, \mathrm{CH}_{2}\right), 1.84 \sim 1.65\left(\mathrm{~m}, 2 \mathrm{H}, \mathrm{CH}_{2}\right), 1.46 \sim 1.36$ (m, 2H, $\mathrm{CH}_{2}$ ), 1.28 (s, 9H, $\mathrm{CH}_{3}$ ), 0.91 (t, $J=7.3 \mathrm{~Hz}, 3 \mathrm{H}$, $\left.\mathrm{CH}_{3}\right) ;{ }^{13} \mathrm{C}$ NMR $\left(75 \mathrm{MHz}, \mathrm{CDCl}_{3}\right) \delta: 162.49,157.68$, $150.60,147.20,134.25,133.14,127.06,126.77,126.38$, $126.20,125.78,120.34,46.21,34.95,34.46,31.25,29.52$, 22.45, 13.76. HRMS (EI) calcd for $\mathrm{C}_{23} \mathrm{H}_{29} \mathrm{~N}_{2} \mathrm{O}[\mathrm{M}+\mathrm{H}]^{+}$ 349.2280, found 349.2284 .

2-丁基-3-(吡啶-3-基甲基)喹唑啉-4(3H)-酮(Q-11): 淡黄色粉末, 收率 77\%. m.p. 100.8 101.4 ${ }^{\circ} \mathrm{C} ;{ }^{1} \mathrm{H}$ NMR $\left(300 \mathrm{MHz}, \mathrm{CDCl}_{3}\right) \delta: 8.62 \sim 8.47(\mathrm{~m}, 1 \mathrm{H}, \mathrm{ArH}), 8.36 \sim$ $8.21(\mathrm{~m}, 1 \mathrm{H}, \mathrm{ArH}), 7.82 \sim 7.56(\mathrm{~m}, 3 \mathrm{H}, \mathrm{ArH}), 7.49 \sim 7.39$ (m, 1H, ArH), $7.28 \sim 7.13(\mathrm{~m}, 2 \mathrm{H}, \operatorname{ArH}), 5.50(\mathrm{~s}, 2 \mathrm{H}$, $\left.\mathrm{CH}_{2}\right), 3.01 \sim 2.71\left(\mathrm{~m}, 2 \mathrm{H}, \mathrm{CH}_{2}\right), 1.88 \sim 1.67\left(\mathrm{~m}, 2 \mathrm{H}, \mathrm{CH}_{2}\right)$, $1.54 \sim 1.32\left(\mathrm{~m}, 2 \mathrm{H}, \mathrm{CH}_{2}\right), 0.92\left(\mathrm{t}, J=7.3 \mathrm{~Hz}, 3 \mathrm{H}, \mathrm{CH}_{3}\right)$; ${ }^{13} \mathrm{C}$ NMR $\left(75 \mathrm{MHz}, \mathrm{CDCl}_{3}\right) \delta: 162.42,157.66,155.85$, $149.41,147.36,136.84,134.20,126.90,126.86,126.26$, 122.53, 121.51, 120.25, 48.24, 35.02, 29.27, 22.36, 13.74. HRMS (EI) calcd for $\mathrm{C}_{18} \mathrm{H}_{20} \mathrm{~N}_{3} \mathrm{O}[\mathrm{M}+\mathrm{H}]^{+}$294.1606, found 294.1605.

2-环丙基-3-(4-氟芐基)喹唑啉-4(3H)-酮(Q-12): 白 色粉末, 收率 86\%. m.p. 108.7 109.1 ${ }^{\circ} \mathrm{C} ;{ }^{1} \mathrm{H}$ NMR (300 $\left.\mathrm{MHz}, \mathrm{CDCl}_{3}\right) \delta: 8.37 \sim 8.21(\mathrm{~m}, 1 \mathrm{H}, \mathrm{ArH}), 7.78 \sim 7.66(\mathrm{~m}$, $1 \mathrm{H}, \mathrm{ArH}), 7.65 \sim 7.53(\mathrm{~m}, 1 \mathrm{H}, \mathrm{ArH}), 7.50 \sim 7.36(\mathrm{~m}, 1 \mathrm{H}$, $\mathrm{ArH}), 7.30 \sim 7.18(\mathrm{~m}, 2 \mathrm{H}, \mathrm{ArH}), 7.07 \sim 6.97(\mathrm{~m}, 2 \mathrm{H}, \mathrm{ArH})$, 5.50 (s, 2H), 1.90 (tt, $J=8.1,4.9 \mathrm{~Hz}, 1 \mathrm{H}, \mathrm{CH}), 1.33 \sim 1.15$ (m, $\left.2 \mathrm{H}, \mathrm{CH}_{2}\right), 1.03 \sim 0.86\left(\mathrm{~m}, 2 \mathrm{H}, \mathrm{CH}_{2}\right) ;{ }^{13} \mathrm{C}$ NMR $(75$ $\left.\mathrm{MHz}, \mathrm{CDCl}_{3}\right) \delta: 163.77,162.58,160.50,157.59,147.56$, $134.25,132.31,132.27,128.33,128.23,126.98,126.96$, 126.15, 120.26, 115.90, 115.61, 45.87, 14.31, 8.92; HRMS (EI) calcd for $\mathrm{C}_{18} \mathrm{H}_{16} \mathrm{FN}_{2} \mathrm{O}[\mathrm{M}+\mathrm{H}]^{+}$295.1247, found 295.1247.

2-环丙基-3-(4-甲氧基芐基)喹唑啉-4(3H)-酮(Q-13): 白色粉末, 收率 78\%. m.p. 106.2 108.2 ${ }^{\circ} \mathrm{C} ;{ }^{1} \mathrm{H}$ NMR $\left(300 \mathrm{MHz}, \mathrm{CDCl}_{3}\right) \delta: 8.35 \sim 8.22(\mathrm{~m}, 1 \mathrm{H}, \mathrm{ArH}), 7.74 \sim$ $7.64(\mathrm{~m}, 1 \mathrm{H}, \mathrm{ArH}), 7.61 \sim 7.53(\mathrm{~m}, 1 \mathrm{H}, \mathrm{ArH}), 7.45 \sim 7.36$ (m, $1 \mathrm{H}, \operatorname{ArH}), 7.24 \sim 7.15(\mathrm{~m}, 2 \mathrm{H}, \mathrm{ArH}), 6.90 \sim 6.80(\mathrm{~m}$, $2 \mathrm{H}, \mathrm{ArH}), 5.52$ (s, $\left.2 \mathrm{H}, \mathrm{CH}_{2}\right), 3.77\left(\mathrm{~s}, 3 \mathrm{H}, \mathrm{CH}_{3}\right), 1.97$ (tt, $J=8.1,4.9 \mathrm{~Hz}, 1 \mathrm{H}, \mathrm{CH}), 1.28 \sim 1.19\left(\mathrm{~m}, 2 \mathrm{H}, \mathrm{CH}_{2}\right), 1.01 \sim$ $0.90\left(\mathrm{~m}, 2 \mathrm{H}, \mathrm{CH}_{2}\right) ;{ }^{13} \mathrm{C} \mathrm{NMR}\left(75 \mathrm{MHz}, \mathrm{CDCl}_{3}\right) \delta: 162.59$, $158.96,157.84,147.57,134.06,128.59,127.88,126.95$, $126.87,125.96,120.34,114.22,55.23,45.96,14.29,8.86$. HRMS (EI) calcd for $\mathrm{C}_{19} \mathrm{H}_{19} \mathrm{~N}_{2} \mathrm{O}_{2}[\mathrm{M}+\mathrm{H}]^{+} 307.1447$, found 307.1444 .

3-(4-氯芐基)-2-环丙基喹唑啉-4(3H)-酮(Q-14)：白 色粉末, 收率 $83 \%$. m.p. 91.3 91.9 ${ }^{\circ} \mathrm{C} ;{ }^{1} \mathrm{H}$ NMR (300 $\left.\mathrm{MHz}, \mathrm{CDCl}_{3}\right) \delta: 8.34 \sim 8.20(\mathrm{~m}, 1 \mathrm{H}, \mathrm{ArH}), 7.81 \sim 7.63(\mathrm{~m}$, $1 \mathrm{H}, \mathrm{ArH}), 7.60 \sim 7.53(\mathrm{~m}, 1 \mathrm{H}, \mathrm{ArH}), 7.47 \sim 7.37(\mathrm{~m}, 1 \mathrm{H}$, ArH), $7.33 \sim 7.24(\mathrm{~m}, 2 \mathrm{H}, \mathrm{ArH}), 7.24 \sim 7.12(\mathrm{~m}, 2 \mathrm{H}), 5.54$ (s, $2 \mathrm{H}, \mathrm{CH}_{2}$ ), 1.86 (tt, $\left.J=8.1,4.9 \mathrm{~Hz}, 1 \mathrm{H}, \mathrm{CH}\right), 1.27 \sim 1.21$ $\left(\mathrm{m}, 2 \mathrm{H}, \mathrm{CH}_{2}\right), 1.01 \sim 0.90\left(\mathrm{~m}, 2 \mathrm{H}, \mathrm{CH}_{2}\right) ;{ }^{13} \mathrm{C}$ NMR $(75$ $\left.\mathrm{MHz}, \mathrm{CDCl}_{3}\right) \delta: 162.47,157.47,147.49,135.03,134.23$, $133.32,128.96,127.88,126.93,126.92,126.12,120.16$, 45.87, 14.26, 8.93. HRMS (EI) calcd for $\mathrm{C}_{18} \mathrm{H}_{16} \mathrm{ClN}_{2} \mathrm{O}$ $[\mathrm{M}+\mathrm{H}]^{+}$311.0951, found 311.095.

3-(4-溴芐基)-2-环丙基喹唑啉-4(3H)-酮(Q-15): 白 色粉末, 收率 81\%. m.p. 106.1 107.2 ${ }^{\circ} \mathrm{C} ;{ }^{1} \mathrm{H}$ NMR (300 $\left.\mathrm{MHz}, \mathrm{CDCl}_{3}\right) \delta: 8.44 \sim 8.09(\mathrm{~m}, 1 \mathrm{H}, \mathrm{ArH}), 7.79 \sim 7.64(\mathrm{~m}$, $1 \mathrm{H}, \mathrm{ArH}), 7.64 \sim 7.53(\mathrm{~m}, 1 \mathrm{H}, \mathrm{ArH}), 7.50 \sim 7.36(\mathrm{~m}, 3 \mathrm{H}$, ArH), $7.18 \sim 7.07(\mathrm{~m}, 2 \mathrm{H}, \mathrm{ArH}), 5.52\left(\mathrm{~s}, 2 \mathrm{H}, \mathrm{CH}_{2}\right), 1.86$ (tt, $J=8.1,4.9 \mathrm{~Hz}, 1 \mathrm{H}, \mathrm{CH}), 1.28 \sim 1.19\left(\mathrm{~m}, 2 \mathrm{H}, \mathrm{CH}_{2}\right)$, $1.01 \sim 0.91\left(\mathrm{~m}, 2 \mathrm{H}, \mathrm{CH}_{2}\right) ;{ }^{13} \mathrm{C}$ NMR $\left(75 \mathrm{MHz}, \mathrm{CDCl}_{3}\right) \delta$ : $162.43,157.43,147.47,135.55,134.20,131.88,128.20$, 126.91, 126.89, 126.10, 121.34, 120.13, 45.91, 14.23, 8.93. HRMS (EI) calcd for $\mathrm{C}_{18} \mathrm{H}_{16} \mathrm{BrN}_{2} \mathrm{O}[\mathrm{M}+\mathrm{H}]^{+} 355.0446$, found 355.0445 .

3-[4-(叔丁基) 芐基]-2-环丙基喹唑啉-4(3H)-酮 (Q-16): 白色粉末，收率 92\%. m.p. 85.7 86.2 ${ }^{\circ} \mathrm{C} ;{ }^{1} \mathrm{H}$ NMR $\left(300 \mathrm{MHz}, \mathrm{CDCl}_{3}\right) \delta: 8.36 \sim 8.19(\mathrm{~m}, 1 \mathrm{H}, \mathrm{ArH})$, $7.76 \sim 7.64(\mathrm{~m}, 1 \mathrm{H}, \operatorname{ArH}), 7.64 \sim 7.53(\mathrm{~m}, 1 \mathrm{H}, \operatorname{ArH})$, $7.46 \sim 7.29$ (m, 3H, ArH), $7.23 \sim 7.12(\mathrm{~m}, 2 \mathrm{H}, \mathrm{ArH}), 5.56$ (s, 2H, $\mathrm{CH}_{2}$ ), 1.97 (tt, $\left.J=8.1,4.9 \mathrm{~Hz}, 1 \mathrm{H}, \mathrm{CH}\right), 1.30$ (s, $\left.9 \mathrm{H}, \mathrm{CH}_{3}\right), 1.27 \sim 1.21\left(\mathrm{~m}, 2 \mathrm{H}, \mathrm{CH}_{2}\right), 1.03 \sim 0.92(\mathrm{~m}, 2 \mathrm{H}$, $\left.\mathrm{CH}_{2}\right) ;{ }^{13} \mathrm{C}$ NMR $\left(75 \mathrm{MHz}, \mathrm{CDCl}_{3}\right) \delta: 162.59,157.90$, $150.45,147.63,134.08,133.42,127.00,126.91,126.22$, $126.00,125.74,120.39,46.24,34.47,31.28,14.37,8.82$. HRMS (EI) calcd for $\mathrm{C}_{22} \mathrm{H}_{25} \mathrm{~N}_{2} \mathrm{O}[\mathrm{M}+\mathrm{H}]^{+} 333.1967$, found 333.1958 .

2-环丙基-3-(2-氟芐基)喹唑啉-4(3H)-酮(Q-17): 白 色粉末, 收率 84\%. m.p. 91.3 92.2 ${ }^{\circ} \mathrm{C} ;{ }^{1} \mathrm{H}$ NMR (300 $\left.\mathrm{MHz}, \mathrm{CDCl}_{3}\right) \delta: 8.38 \sim 8.14(\mathrm{~m}, 1 \mathrm{H}), 7.77 \sim 7.64(\mathrm{~m}, 1 \mathrm{H}$, ArH), $7.64 \sim 7.49(\mathrm{~m}, 1 \mathrm{H}, \mathrm{ArH}), 7.47 \sim 7.36(\mathrm{~m}, 1 \mathrm{H}, \mathrm{ArH})$, $7.29 \sim 7.16(\mathrm{~m}, 1 \mathrm{H}, \mathrm{ArH}), 7.12 \sim 6.99(\mathrm{~m}, 3 \mathrm{H}, \mathrm{ArH}), 5.64$ (s, $2 \mathrm{H}, \mathrm{CH}_{2}$ ), 1.88 (tt, $\left.J=8.1,4.9 \mathrm{~Hz}, 1 \mathrm{H}, \mathrm{CH}\right), 1.25 \sim 1.15$ 
$\left(\mathrm{m}, 2 \mathrm{H}, \mathrm{CH}_{2}\right), 1.00 \sim 0.89\left(\mathrm{~m}, 2 \mathrm{H}, \mathrm{CH}_{2}\right) ;{ }^{13} \mathrm{C}$ NMR $(75$ $\left.\mathrm{MHz}, \mathrm{CDCl}_{3}\right) \delta: 162.52,161.64,158.38,157.54,147.48$, $134.13,129.08,128.97,127.84,127.80,126.87,126.02$, $124.53,124.48,123.68,123.49,120.11,115.41,115.13$, 77.42, 77.00, 76.58, 40.13, 40.06, 13.97, 13.95, 8.79. HRMS (EI) calcd for $\mathrm{C}_{18} \mathrm{H}_{16} \mathrm{FN}_{2} \mathrm{O}[\mathrm{M}+\mathrm{H}]^{+}$295.1247, found 295.1246 .

3-(3-氯芐基)-2-戊基喹唑啉-4(3H)-酮(Q-18): 白色 固体, 产率 $75.19 \%$. m.p. $86.7 \sim 88.1{ }^{\circ} \mathrm{C} ;{ }^{1} \mathrm{H}$ NMR $(300$ $\left.\mathrm{MHz}, \mathrm{CDCl}_{3}\right) \delta: 8.39 \sim 8.20(\mathrm{~m}, 1 \mathrm{H}, \mathrm{ArH}), 7.76 \sim 7.61(\mathrm{~m}$, $2 \mathrm{H}, \mathrm{ArH}), 7.49 \sim 7.36(\mathrm{~m}, 1 \mathrm{H}, \mathrm{ArH}), 7.23 \sim 7.11(\mathrm{~m}, 2 \mathrm{H}$, $\mathrm{ArH}), 7.04 \sim 6.94(\mathrm{~m}, 2 \mathrm{H}, \mathrm{ArH}), 5.36\left(\mathrm{~s}, 2 \mathrm{H}, \mathrm{CH}_{2}\right), 2.82 \sim$ $2.63\left(\mathrm{~m}, 2 \mathrm{H}, \mathrm{CH}_{2}\right), 1.88 \sim 1.62\left(\mathrm{~m}, 2 \mathrm{H}, \mathrm{CH}_{2}\right), 1.46 \sim 1.21$ (m, $\left.4 \mathrm{H}, \mathrm{CH}_{2}\right), 0.88\left(\mathrm{t}, J=7.0 \mathrm{~Hz}, 3 \mathrm{H}, \mathrm{CH}_{3}\right) ;{ }^{13} \mathrm{C}$ NMR $(75$ $\left.\mathrm{MHz}, \mathrm{CDCl}_{3}\right) \delta: 162.16,156.71,147.13,138.23,134.63$, $134.12,129.94,127.61,126.84,126.77,126.35,126.24$, 124.34, 120.02, 45.67, 34.85, 31.17, 26.59, 22.16, 13.71. HRMS (EI) calcd for $\mathrm{C}_{20} \mathrm{H}_{22} \mathrm{ClN}_{2} \mathrm{O}[\mathrm{M}+\mathrm{H}]^{+} 341.1421$, found 341.1411 .

3-(4-氟芐基)-2-戊基喹唑啉-4(3H)-酮(Q-19): 白色 固体, 收率 81\%. m.p. 96.7 97.9 ${ }^{\circ} \mathrm{C} ;{ }^{1} \mathrm{H}$ NMR (300 $\left.\mathrm{MHz}, \mathrm{CDCl}_{3}\right) \delta: 8.36 \sim 8.21(\mathrm{~m}, 1 \mathrm{H}, \mathrm{ArH}), 7.79 \sim 7.60(\mathrm{~m}$, $2 \mathrm{H}, \mathrm{ArH}), 7.48 \sim 7.35(\mathrm{~m}, 1 \mathrm{H}, \mathrm{ArH}), 7.25 \sim 7.15(\mathrm{~m}, 3 \mathrm{H}$, $\mathrm{ArH}), 7.10 \sim 6.99(\mathrm{~m}, 1 \mathrm{H}, \mathrm{ArH}), 5.36\left(\mathrm{~s}, 2 \mathrm{H}, \mathrm{CH}_{2}\right), 2.79 \sim$ $2.59\left(\mathrm{~m}, 2 \mathrm{H}, \mathrm{CH}_{2}\right), 1.90 \sim 1.63\left(\mathrm{~m}, 2 \mathrm{H}, \mathrm{CH}_{2}\right), 1.39 \sim 1.24$ (m, 4H, $\left.\mathrm{CH}_{2}\right), 0.87$ (t, $\left.J=7.1 \mathrm{~Hz}, 3 \mathrm{H}, \mathrm{CH}_{3}\right) ;{ }^{13} \mathrm{C}$ NMR $(75$ $\left.\mathrm{MHz}, \mathrm{CDCl}_{3}\right) \delta: 163.56,162.28,160.30,156.86,147.18$, $134.07,131.96,131.92,128.11,128.00,126.82,126.75$, 126.20, 120.11, 115.71, 115.42, 45.57, 34.89, 31.24, 26.63, 22.17, 13.71; HRMS (EI) calcd for $\mathrm{C}_{20} \mathrm{H}_{22} \mathrm{FN}_{2} \mathrm{O}[\mathrm{M}+\mathrm{H}]^{+}$ 325.1716, found 325.1718 .

3-(3-氟苄基)-2-戊基喹唑啉-4(3H)-酮(Q-20): 白色 固体, 收率 $89 \%$. m.p. 100.2 101.2 ${ }^{\circ} \mathrm{C} ;{ }^{1} \mathrm{H}$ NMR (300 $\left.\mathrm{MHz}, \mathrm{CDCl}_{3}\right) \delta: 8.38 \sim 8.23(\mathrm{~m}, 1 \mathrm{H}, \mathrm{ArH}), 7.79 \sim 7.60(\mathrm{~m}$, $2 \mathrm{H}, \mathrm{ArH}), 7.51 \sim 7.39(\mathrm{~m}, 1 \mathrm{H}, \mathrm{ArH}), 7.33 \sim 7.18(\mathrm{~m}, 1 \mathrm{H}$, $\mathrm{ArH}), 7.02 \sim 6.84(\mathrm{~m}, 3 \mathrm{H}, \mathrm{ArH}), 5.39\left(\mathrm{~s}, 2 \mathrm{H}, \mathrm{CH}_{2}\right), 2.79 \sim$ $2.65\left(\mathrm{~m}, 2 \mathrm{H}, \mathrm{CH}_{2}\right), 1.87 \sim 1.69\left(\mathrm{~m}, 2 \mathrm{H}, \mathrm{CH}_{2}\right), 1.41 \sim 1.22$ (m, 4H, $\left.\mathrm{CH}_{2}\right), 0.87$ (t, $\left.J=7.1 \mathrm{~Hz}, 3 \mathrm{H}, \mathrm{CH}_{3}\right) ;{ }^{13} \mathrm{C}$ NMR $(75$ $\left.\mathrm{MHz}, \mathrm{CDCl}_{3}\right) \delta: 164.59,162.25,161.32,156.83,147.19$, $138.83,138.73,134.18,130.38,130.27,126.88,126.82$, $126.30,121.80,121.77,120.07,114.54,114.26,113.47$, 113.17, 45.78, 45.75, 34.90, 31.24, 26.66, 22.19, 13.73. HRMS (EI) calcd for $\mathrm{C}_{20} \mathrm{H}_{22} \mathrm{FN}_{2} \mathrm{O}[\mathrm{M}+\mathrm{H}]^{+} 325.1716$, found 325.1718 .

3-(2-氟苄基)-2-戊基喹唑啉-4(3H)-酮(Q-21)：白色
固体, 收率 88\%. m.p. 82.3 84.6 ${ }^{\circ} \mathrm{C} ;{ }^{1} \mathrm{H}$ NMR (300 $\left.\mathrm{MHz}, \mathrm{CDCl}_{3}\right) \delta: 8.37 \sim 8.22(\mathrm{~m}, 1 \mathrm{H}, \mathrm{ArH}), 7.83 \sim 7.61(\mathrm{~m}$, $2 \mathrm{H}, \mathrm{ArH}), 7.49 \sim 7.36(\mathrm{~m}, 1 \mathrm{H}, \mathrm{ArH}), 7.27 \sim 7.18(\mathrm{~m}, 1 \mathrm{H}$, $\mathrm{ArH}), 7.13 \sim 6.93(\mathrm{~m}, 3 \mathrm{H}, \mathrm{ArH}), 5.45\left(\mathrm{~s}, 2 \mathrm{H}, \mathrm{CH}_{2}\right), 2.88 \sim$ $2.62\left(\mathrm{~m}, 2 \mathrm{H}, \mathrm{CH}_{2}\right), 1.93 \sim 1.62\left(\mathrm{~m}, 2 \mathrm{H}, \mathrm{CH}_{2}\right), 1.45 \sim 1.21$ (m, 4H, $\left.\mathrm{CH}_{2}\right), 0.87$ (t, $\left.J=7.0 \mathrm{~Hz}, 3 \mathrm{H}, \mathrm{CH}_{3}\right) ;{ }^{13} \mathrm{C}$ NMR $(75$ $\left.\mathrm{MHz}, \mathrm{CDCl}_{3}\right) \delta: 162.41,161.55,158.29,157.00,147.24$, $134.14,129.12,129.01,127.74,127.69,126.86,126.82$, $126.25,124.49,124.44,123.39,123.21,120.10,115.41$, 115.13, 40.04, 39.97, 34.73, 31.24, 26.78, 22.17, 13.74 . HRMS (EI) calcd for $\mathrm{C}_{20} \mathrm{H}_{22} \mathrm{FN}_{2} \mathrm{O}[\mathrm{M}+\mathrm{H}]^{+} 325.1716$, found 325.1717 .

2-丁基-3-(3-氯芐基)喹唑啉-4(3H)-酮(Q-22): 白色 固体, 收率 71\%. m.p. 96.9 98.0 ${ }^{\circ} \mathrm{C} ;{ }^{1} \mathrm{H}$ NMR (300 $\left.\mathrm{MHz}, \mathrm{CDCl}_{3}\right) \delta: 8.39 \sim 8.21(\mathrm{~m}, 1 \mathrm{H}), 7.74 \sim 7.60(\mathrm{~m}, 2 \mathrm{H}$, ArH), $7.45 \sim 7.32(\mathrm{~m}, 1 \mathrm{H}, \mathrm{ArH}), 7.26 \sim 7.21(\mathrm{~m}, 2 \mathrm{H}, \mathrm{ArH})$, $7.18 \sim 7.11(\mathrm{~m}, 1 \mathrm{H}, \mathrm{ArH}), 7.10 \sim 6.99(\mathrm{~m}, 1 \mathrm{H}, \mathrm{ArH}), 5.37$ (s, $\left.2 \mathrm{H}, \mathrm{CH}_{2}\right), 2.79 \sim 2.64\left(\mathrm{~m}, 2 \mathrm{H}, \mathrm{CH}_{2}\right), 1.86 \sim 1.67(\mathrm{~m}$, $\left.2 \mathrm{H}, \mathrm{CH}_{2}\right), 1.52 \sim 1.26\left(\mathrm{~m}, 2 \mathrm{H}, \mathrm{CH}_{2}\right), 0.91(\mathrm{t}, J=7.3 \mathrm{~Hz}$, $\left.3 \mathrm{H}, \mathrm{CH}_{3}\right) ;{ }^{13} \mathrm{C}$ NMR $\left(75 \mathrm{MHz}, \mathrm{CDCl}_{3}\right) \delta: 162.27,156.81$, $147.19,138.26,134.74,134.24,130.04,127.72,126.90$, $126.87,126.42,126.37,124.40,120.09,45.76,34.72$, 29.08, 22.24, 13.64. HRMS (EI) calcd for $\mathrm{C}_{19} \mathrm{H}_{20} \mathrm{ClN}_{2} \mathrm{O}$ $[\mathrm{M}+\mathrm{H}]^{+}$327.1264, found 327.1265.

2-丁基-3-(4-氟芐基)喹唑啉-4(3H)-酮(Q-23)：白色 固体, 收率 83\%. m.p. 106.5 108.4 ${ }^{\circ} \mathrm{C} ;{ }^{1} \mathrm{H}$ NMR (300 $\left.\mathrm{MHz}, \mathrm{CDCl}_{3}\right) \delta: 8.37 \sim 8.21(\mathrm{~m}, 1 \mathrm{H}, \mathrm{ArH}), 7.80 \sim 7.59(\mathrm{~m}$, $2 \mathrm{H}, \operatorname{ArH}), 7.49 \sim 7.39(\mathrm{~m}, 1 \mathrm{H}, \mathrm{ArH}), 7.21 \sim 7.10(\mathrm{~m}, 2 \mathrm{H}$, $\operatorname{ArH}), 7.07 \sim 6.92(\mathrm{~m}, 2 \mathrm{H}, \mathrm{ArH}), 5.36\left(\mathrm{~s}, 2 \mathrm{H}, \mathrm{CH}_{2}\right), 2.84 \sim$ $2.61\left(\mathrm{~m}, 2 \mathrm{H}, \mathrm{CH}_{2}\right), 1.86 \sim 1.66\left(\mathrm{~m}, 2 \mathrm{H}, \mathrm{CH}_{2}\right), 1.49 \sim 1.29$ (m, $\left.2 \mathrm{H}, \mathrm{CH}_{2}\right), 0.91\left(\mathrm{t}, J=7.3 \mathrm{~Hz}, 3 \mathrm{H}, \mathrm{CH}_{3}\right) ;{ }^{13} \mathrm{C}$ NMR $(75$ $\left.\mathrm{MHz}, \mathrm{CDCl}_{3}\right) \delta: 163.63,162.36,160.37,156.93,147.23$, $134.16,131.99,131.95,128.15,128.05,126.87,126.83$, 126.29, 120.17, 115.78, 115.50, 45.64, 34.72, 29.08, 22.26, 13.63. HRMS (EI) calcd for $\mathrm{C}_{19} \mathrm{H}_{20} \mathrm{FN}_{2} \mathrm{O}[\mathrm{M}+\mathrm{H}]^{+}$ 311.1560 , found 311.1561 .

2-丁基-3-(3-氟芐基)喹唑啉-4(3H)-酮(Q-24)：白色 固体, 收率 91\%. m.p. 80.7 82.5 ${ }^{\circ} \mathrm{C} ;{ }^{1} \mathrm{H}$ NMR (300 $\left.\mathrm{MHz}, \mathrm{CDCl}_{3}\right) \delta: 8.35 \sim 8.24(\mathrm{~m}, 1 \mathrm{H}, \mathrm{ArH}), 7.79 \sim 7.59(\mathrm{~m}$, $2 \mathrm{H}, \mathrm{ArH}), 7.50 \sim 7.38(\mathrm{~m}, 1 \mathrm{H}, \mathrm{ArH}), 7.34 \sim 7.20(\mathrm{~m}, 1 \mathrm{H}$, $\mathrm{ArH}), 7.01 \sim 6.84(\mathrm{~m}, 3 \mathrm{H}, \mathrm{ArH}), 5.39\left(\mathrm{~s}, 2 \mathrm{H}, \mathrm{CH}_{2}\right), 2.72 \sim$ $2.59\left(\mathrm{~m}, 2 \mathrm{H}, \mathrm{CH}_{2}\right), 1.84 \sim 1.68\left(\mathrm{~m}, 2 \mathrm{H}, \mathrm{CH}_{2}\right), 1.51 \sim 1.30$ (m, $\left.2 \mathrm{H}, \mathrm{CH}_{2}\right), 0.90$ (t, $\left.J=7.3 \mathrm{~Hz}, 3 \mathrm{H}, \mathrm{CH}_{3}\right) ;{ }^{13} \mathrm{C}$ NMR $(75$ $\left.\mathrm{MHz}, \mathrm{CDCl}_{3}\right) \delta: 164.59,162.25,161.31,156.80,147.20$, $138.83,138.73,134.17,130.38,130.27,126.88,126.82$, 
$126.30,121.80,121.76,120.07,114.53,114.25,113.46$, 113.16, 45.77, 45.74, 34.64, 29.01, 22.20, 14.00, 13.58 . HRMS (EI) calcd for $\mathrm{C}_{19} \mathrm{H}_{20} \mathrm{FN}_{2} \mathrm{O}[\mathrm{M}+\mathrm{H}]^{+} 311.1560$, found 311.1559 .

2-丁基-3-(2-氟苠基)喹唑啉-4(3H)-酥(Q-25): 白色 粉末, 收率 87\%. m.p. 85.6 87.2 ${ }^{\circ} \mathrm{C} ;{ }^{1} \mathrm{H}$ NMR (300 $\left.\mathrm{MHz} \mathrm{CDCl}_{3}\right) \delta: 8.31 \sim 8.27(\mathrm{~m}, 1 \mathrm{H}, \mathrm{ArH}), 7.73 \sim 7.67(\mathrm{~m}$, $2 \mathrm{H}, \mathrm{ArH}), 7.46 \sim 7.43(\mathrm{~m}, 1 \mathrm{H}, \mathrm{ArH}), 7.12 \sim 7.10(\mathrm{~m}, 1 \mathrm{H}$, $\mathrm{ArH}), 7.08 \sim 6.99(\mathrm{~m}, 3 \mathrm{H}, \mathrm{ArH}), 5.46\left(\mathrm{~s}, 2 \mathrm{H}, \mathrm{CH}_{2}\right), 2.73 \sim$ $2.60\left(\mathrm{~m}, 2 \mathrm{H}, \mathrm{CH}_{2}\right), 1.81 \sim 1.69\left(\mathrm{~m}, 2 \mathrm{H}, \mathrm{CH}_{2}\right), 1.44 \sim 1.38$ $\left(\mathrm{m}, 2 \mathrm{H}, \mathrm{CH}_{2}\right), 0.91\left(\mathrm{t}, J=7.3 \mathrm{~Hz}, 3 \mathrm{H}, \mathrm{CH}_{3}\right) ;{ }^{13} \mathrm{C} \mathrm{NMR}(75$ $\left.\mathrm{MHz}, \mathrm{CDCl}_{3}\right) \delta: 162.48,161.59,158.34,157.05,147.27$, $134.21,129.18,129.07,127.77,127.72,126.90,126.87$, 126.32 , 124.54, 124.49, 123.42, 123.23, 120.14, 115.46, $115.18,40.09$, 40.02, 34.54, 29.21, 22.26, 13.60. HRMS (EI) calcd for $\mathrm{C}_{19} \mathrm{H}_{20} \mathrm{FN}_{2} \mathrm{O}[\mathrm{M}+\mathrm{H}]^{+} 311.1560$, found 311.1559 .

2-丁基-3-(3-氯苠基)-8-甲基喹唑啉-4(3H)-酠(Q-26): 白色固体, 收率 82\%. m.p. 88.1 88.9 ${ }^{\circ} \mathrm{C} ;{ }^{1} \mathrm{H}$ NMR $(300$ MHz, DMSO- $\left.d_{6}\right) \delta: 8.22 \sim 8.06(\mathrm{~m}, 1 \mathrm{H}, \mathrm{ArH}), 7.59 \sim 7.46$ $(\mathrm{m}, 1 \mathrm{H}, \mathrm{ArH}), 7.39 \sim 7.30(\mathrm{~m}, 1 \mathrm{H}, \mathrm{ArH}), 7.26 \sim 7.21(\mathrm{~m}$, $2 \mathrm{H}, \mathrm{ArH}), 7.19 \sim 7.14(\mathrm{~m}, 1 \mathrm{H}, \mathrm{ArH}), 7.09 \sim 7.00(\mathrm{~m}, 1 \mathrm{H}$, $\mathrm{ArH}), 5.36\left(\mathrm{~s}, 2 \mathrm{H}, \mathrm{CH}_{2}\right), 2.79 \sim 2.64\left(\mathrm{~m}, 2 \mathrm{H}, \mathrm{CH}_{2}\right), 2.61(\mathrm{~s}$, $\left.3 \mathrm{H}, \mathrm{CH}_{3}\right), 1.90 \sim 1.73\left(\mathrm{~m}, 2 \mathrm{H}, \mathrm{CH}_{2}\right), 1.52 \sim 1.32(\mathrm{~m}, 2 \mathrm{H}$, $\mathrm{CH}_{2}$ ), $0.92\left(\mathrm{t}, J=7.3 \mathrm{~Hz}, 3 \mathrm{H}, \mathrm{CH}_{3}\right) ;{ }^{13} \mathrm{C} \mathrm{NMR}(75 \mathrm{MHz}$, $\left.\mathrm{CDCl}_{3}\right) \delta: 162.86,155.03,145.79,138.47,135.72,134.84$, $134.83,130.11,127.78,126.54,126.02,124.61,124.54$, $120.12,45.68,34.47,28.52,22.15,17.06,13.83$. HRMS (EI) calcd for $\mathrm{C}_{20} \mathrm{H}_{22} \mathrm{ClN}_{2} \mathrm{O}[\mathrm{M}+\mathrm{H}]^{+} 341.1421$, found 341.1422 .

2-丁基-3-(4-氟苠基)-8-甲基喹唑啉-4(3H)-酮(Q-27): 白色固体, 收率 82\%. m.p. 89.0 92.0 ${ }^{\circ} \mathrm{C} ;{ }^{1} \mathrm{H}$ NMR $(300$ MHz, DMSO- $\left.d_{6}\right) \delta: 8.23 \sim 8.07(\mathrm{~m}, 1 \mathrm{H}, \mathrm{ArH}), 7.65 \sim 7.51$ $(\mathrm{m}, 1 \mathrm{H}, \mathrm{ArH}), 7.38 \sim 7.28(\mathrm{~m}, 1 \mathrm{H}, \mathrm{ArH}), 7.22 \sim 7.12(\mathrm{~m}$, $2 \mathrm{H}, \mathrm{ArH}), 7.06 \sim 6.94(\mathrm{~m}, 2 \mathrm{H}, \mathrm{ArH}), 5.35\left(\mathrm{~s}, 2 \mathrm{H}, \mathrm{CH}_{2}\right)$, $2.79 \sim 2.65\left(\mathrm{~m}, 2 \mathrm{H}, \mathrm{CH}_{2}\right), 2.60\left(\mathrm{~s}, 3 \mathrm{H}, \mathrm{CH}_{3}\right), 1.88 \sim 1.74$ $\left(\mathrm{m}, 2 \mathrm{H}, \mathrm{CH}_{2}\right), 1.51 \sim 1.33\left(\mathrm{~m}, 2 \mathrm{H}, \mathrm{CH}_{2}\right), 0.92(\mathrm{t}, J=7.3$ $\left.\mathrm{Hz}, 3 \mathrm{H}, \mathrm{CH}_{3}\right) ;{ }^{13} \mathrm{C}$ NMR $\left(75 \mathrm{MHz}, \mathrm{CDCl}_{3}\right) \delta: 163.74$, $162.93,160.47,155.16,145.80,135.67,134.74,132.18$, $132.14,128.28,128.18,125.95,124.56,120.19,115.86$, $115.58,45.55,34.46,28.51,22.17,17.04,13.82$. HRMS (EI) calcd for $\mathrm{C}_{20} \mathrm{H}_{22} \mathrm{FN}_{2} \mathrm{O}[\mathrm{M}+\mathrm{H}]^{+} 325.1716$, found 325.1715 .

2-丁基-3-(3-氟苠基)-8-甲基喹唑啉-4(3H)-酩(Q-28): 白色固体, 收率 78\%. m.p. 83.5 84.9 ${ }^{\circ} \mathrm{C} ;{ }^{1} \mathrm{H}$ NMR (300
MHz, DMSO- $\left.d_{6}\right) \delta: 8.24 \sim 8.05(\mathrm{~m}, 1 \mathrm{H}, \mathrm{ArH}), 7.59 \sim 7.43$ $(\mathrm{m}, 1 \mathrm{H}, \mathrm{ArH}), 7.39 \sim 7.22(\mathrm{~m}, 2 \mathrm{H}, \mathrm{ArH}), 7.09 \sim 6.74(\mathrm{~m}$, $3 \mathrm{H}, \mathrm{ArH}), 5.38\left(\mathrm{~s}, 2 \mathrm{H}, \mathrm{CH}_{2}\right), 2.78 \sim 2.66\left(\mathrm{~m}, 2 \mathrm{H}, \mathrm{CH}_{2}\right)$, $2.61\left(\mathrm{~s}, 3 \mathrm{H}, \mathrm{CH}_{3}\right), 1.91 \sim 1.71\left(\mathrm{~m}, 2 \mathrm{H}, \mathrm{CH}_{2}\right), 1.52 \sim 1.30$ $\left(\mathrm{m}, 2 \mathrm{H}, \mathrm{CH}_{2}\right), 0.92\left(\mathrm{t}, J=7.3 \mathrm{~Hz}, 3 \mathrm{H}, \mathrm{CH}_{3}\right) ;{ }^{13} \mathrm{C} \mathrm{NMR}(75$ $\left.\mathrm{MHz}, \mathrm{CDCl}_{3}\right) \delta: 164.76,162.86,161.48,155.07,145.80$, $139.04,138.95,135.71,134.81,130.48,130.37,126.00$, $124.60,121.97,121.93,120.13,114.62,114.34,113.61$, $113.31,77.42,77.00,76.58,45.73,45.70,34.43,28.50$, 22.15, 17.05, 13.81. HRMS (EI) calcd for $\mathrm{C}_{20} \mathrm{H}_{22} \mathrm{FN}_{2} \mathrm{O}$ $[\mathrm{M}+\mathrm{H}]^{+}$325.1716, found 325.1719.

6-氯-2-环己基-3-(4-氟芐基)喹唑啉-4(3H)-酮(Q-29): 白色固体，收率 $80 \%$. m.p. 126.0 $127.4{ }^{\circ} \mathrm{C} ;{ }^{1} \mathrm{H}$ NMR $\left(300 \mathrm{MHz}, \mathrm{CDCl}_{3}\right) \delta: 8.24(\mathrm{~d}, J=2.3 \mathrm{~Hz}, 1 \mathrm{H}, \mathrm{ArH})$, $7.71 \sim 7.53(\mathrm{~m}, 2 \mathrm{H}, \mathrm{ArH}), 7.24 \sim 7.10(\mathrm{~m}, 2 \mathrm{H}, \mathrm{ArH})$, $7.09 \sim 6.93(\mathrm{~m}, 2 \mathrm{H}, \mathrm{ArH}), 5.38\left(\mathrm{~s}, 2 \mathrm{H}, \mathrm{CH}_{2}\right), 2.91 \sim 2.57$ $(\mathrm{m}, 1 \mathrm{H}, \mathrm{CH}), 1.91 \sim 1.58\left(\mathrm{~m}, 7 \mathrm{H}, \mathrm{CH}_{2}\right), 1.41 \sim 1.07(\mathrm{~m}$, $\left.3 \mathrm{H}, \mathrm{CH}_{2}\right) ;{ }^{13} \mathrm{C} \mathrm{NMR}\left(75 \mathrm{MHz}, \mathrm{CDCl}_{3}\right) \delta: 163.76,161.69$, $160.91,160.49,146.03,134.56,132.30,132.26,131.93$, $128.83,128.25,128.14,126.19,121.26,115.95,115.67$, 45.51, 42.47, 31.45, 26.01, 25.52. HRMS (EI) calcd for $\mathrm{C}_{21} \mathrm{H}_{21} \mathrm{ClFN}_{2} \mathrm{O}[\mathrm{M}+\mathrm{H}]^{+}$371.1326, found 371.1322.

6-氯-2-环己基-3-(2-氟苠基)喹唑啉-4(3H)-酤(Q-30): 白色固体, 收率 $72 \%$. m.p. 122.1 123.6 ${ }^{\circ} \mathrm{C} ;{ }^{1} \mathrm{H}$ NMR $\left(300 \mathrm{MHz}, \mathrm{DMSO}-d_{6}\right) \delta: 8.26 \sim 8.11(\mathrm{~m}, 1 \mathrm{H}, \mathrm{ArH}), 7.72 \sim$ $7.52(\mathrm{~m}, 2 \mathrm{H}, \mathrm{ArH}), 7.31 \sim 7.20(\mathrm{~m}, 1 \mathrm{H}, \mathrm{ArH}), 7.17 \sim 6.91$ (m, 3H, ArH), $5.47\left(\mathrm{~s}, 2 \mathrm{H}, \mathrm{CH}_{2}\right), 2.81 \sim 2.58(\mathrm{~m}, 1 \mathrm{H}, \mathrm{CH})$, $1.88 \sim 1.60\left(\mathrm{~m}, 7 \mathrm{H}, \mathrm{CH}_{2}\right), 1.39 \sim 1.10\left(\mathrm{~m}, 3 \mathrm{H}, \mathrm{CH}_{2}\right) ;{ }^{13} \mathrm{C}$ NMR (75 MHz, $\left.\mathrm{CDCl}_{3}\right) \delta: 161.75,161.52,160.92,158.26$, $146.02,134.52,131.89,129.37,129.27,128.81,128.01$, $127.96,126.16,124.63,124.58,123.69,123.50,121.19$, 115.56, 115.28, 42.14, 42.13, 39.76, 39.69, 31.48, 25.89, 25.55. HRMS (EI) calcd for $\mathrm{C}_{21} \mathrm{H}_{21} \mathrm{ClFN}_{2} \mathrm{O}[\mathrm{M}+\mathrm{H}]^{+}$ 371.1326 , found 371.1322 .

6-氯-2-环己基-3-(3-氟苠基)喹唑啉-4(3H)-酮(Q-31): 白色固体，收率 78\%. m.p. 98.2 99.4 ${ }^{\circ} \mathrm{C} ;{ }^{1} \mathrm{H}$ NMR (300 $\left.\mathrm{MHz} \mathrm{CDCl}_{3}\right) \delta: 8.25 \sim 8.11(\mathrm{~m}, 1 \mathrm{H}, \mathrm{ArH}), 7.70 \sim 7.52(\mathrm{~m}$, $2 \mathrm{H}, \mathrm{ArH}), 7.37 \sim 7.23(\mathrm{~m}, 1 \mathrm{H}, \mathrm{ArH}), 7.03 \sim 6.92(\mathrm{~m}, 2 \mathrm{H}$, $\mathrm{ArH}), 6.91 \sim 6.84(\mathrm{~m}, 1 \mathrm{H}, \mathrm{ArH}), 5.41\left(\mathrm{~s}, 2 \mathrm{H}, \mathrm{CH}_{2}\right), 2.75 \sim$ $2.63(\mathrm{~m}, 1 \mathrm{H}, \mathrm{CH}), 1.94 \sim 1.61\left(\mathrm{~m}, 7 \mathrm{H}, \mathrm{CH}_{2}\right), 1.40 \sim 1.09$ $\left(\mathrm{m}, 3 \mathrm{H}, \mathrm{CH}_{2}\right) ;{ }^{13} \mathrm{C} \mathrm{NMR}\left(75 \mathrm{MHz}, \mathrm{CDCl}_{3}\right) \delta$ : 164.64, $161.53,161.36,160.77,145.97,139.08,138.99,134.52$, $131.89,130.49,130.38,128.81,126.13,121.88,121.84$, $121.15,114.73,114.45,113.55,113.25,45.62,45.60$, 42.41, 31.40, 25.91, 25.47. HRMS (EI) calcd for $\mathrm{C}_{21} \mathrm{H}_{21} \mathrm{Cl}$ - 
$\mathrm{FN}_{2} \mathrm{O}[\mathrm{M}+\mathrm{H}]^{+} 371.1326$, found 371.1318 .

6-氯-3-苠氨基-2-新戊基-8-甲基喹唑啉-4(3H)-酠: m.p. $127.3 \sim 127.9{ }^{\circ} \mathrm{C}$; ${ }^{1} \mathrm{H}$ NMR (300 MHz, $\left.\mathrm{CDCl}_{3}\right) \delta$ : 7.65 (d, $J=2.3 \mathrm{~Hz}, 1 \mathrm{H}, \mathrm{Ar}-\mathrm{H}), 7.49 \sim 7.43$ (m, 1H, Ar-H), $7.40 \sim 7.22(\mathrm{~m}, 3 \mathrm{H}, \mathrm{Ar}-\mathrm{H}), 7.21 \sim 7.15(\mathrm{~m}, 1 \mathrm{H}, \mathrm{Ar}-\mathrm{H})$, $7.15 \sim 7.08(\mathrm{~m}, 1 \mathrm{H}, \mathrm{Ar}-\mathrm{H}), 5.52(\mathrm{~s}, 1 \mathrm{H}, \mathrm{NH}), 3.85$ (s, 2H, Ar- $\left.\mathrm{CH}_{2}\right), 2.20$ (s, 3H, Ar- $\left.\mathrm{CH}_{3}\right), 2.09$ (s, 2H, $\left.\mathrm{CH}_{2} \mathrm{C}\right), 1.03$ [s, 9H, $\left.\mathrm{C}\left(\mathrm{CH}_{3}\right)_{3}\right]$. HRMS (EI) calcd for $\mathrm{C}_{21} \mathrm{H}_{25} \mathrm{ClN}_{3} \mathrm{O}$, $[\mathrm{M}+\mathrm{H}]^{+}$370.1686, found 370.1688 .

\section{3 生物活性测试}

试虫包括: 小菜蛾(Plutella xylostella Linnaeus)、棉 蚜(Aphis gossypii Glover).

抗肿瘤测试细胞包括: 人肺癌细胞(A549 细胞)、人 肝癌细胞(Bel7402 细胞)、人结肠腺癌细胞(HCT-8 细胞).

离体杀菌活性测试菌种包括: 黄瓜枯萎病原黄瓜尖 镰孢菌(Fusarium oxysporum sp. cucmrium)、花生褐斑病 原落花生尾狍(Cercospora arachidicola)、苹果轮纹病原 菌(Botryospuaeria berengeriana)、番茄早疫病原茄链格 孢菌(Alternaria tenuis Nees)、小麦赤霉病原和谷镰刀菌 (Fusarium graminearum)、辣椒疫霉病原疫霉菌 (Phytophthora capsici Leonian)、油菜菌核病原核盘菌 (Sclerotinia sclerotiorum)、黄瓜灰霉病原灰葡萄菌 (Botrytis cinerea Pers.)、水稻纹枯病原水稻纹枯菌 (Rhizoctonia solani) 和马铃薯晚疫病原致病疫霉菌 (Phytophthora infestans).

活体杀菌活性测试菌种包括: 辣椒疫霉病原疫霉菌 (Phytophthora capsici Leonian)在辣椒幼苗上进行测试; 小麦白粉病原布氏白粉菌(Blumeria graminis)在小麦幼 苗上进行测试.

\subsection{6 -氯-3-芳氨基-2-新戊基-8-甲基喹唑啉-4(3H)-酮} 的物化数据、晶体结构解析及结果

单晶 $\mathrm{X}$ 射线衍射实验测定的化合物化学式为: $\mathrm{C}_{21} \mathrm{H}_{24} \mathrm{ClN}_{3} \mathrm{O}$, 分子质量为: 369.88 (计算值为 369.89); 该 化合物晶体为三斜晶系, 晶胞参数: $a=0.9315(3) \mathrm{nm}$, $\alpha=75.456(12)^{\circ}, b=1.0572(3) \mathrm{nm}, \beta=71.236(9)^{\circ}, c=$ 1.0854(3) nm, $\gamma=88.278(13)^{\circ}$, 晶胞体积为: 0.9781(4) $\mathrm{nm}^{3}$, 晶胞中分子数: $Z=2, F(000)=392$. 计算密度为: $1256 \mathrm{~kg} \cdot \mathrm{m}^{-3}$. 所用 $\mathrm{X}$ 射线波长 $\lambda=0.071073 \mathrm{~nm}$, 吸收系 数 $\mu=0.210 \mathrm{~mm}^{-1}$, 共收集到 10900 个衍射点, 3428 个独 立衍射点 $\left(R_{\mathrm{int}}=0.0486\right)$, 其中 3155 个可观测点 $[I>2 \sigma(I)]$ 用于晶体结构解析, 最终差值残余电子密度的最高峰 为: $624 \mathrm{e} \cdot \mathrm{nm}^{-3}$, 最低峰为: $-587 \mathrm{e} \cdot \mathrm{nm}^{-3}$. 晶体结构用 SHELXL-97 程序对 $F^{2}$ 进行精修 ${ }^{[20]}$ 获得非氢原子坐标及 各向异性参数, 氢原子由差值 Fourier 合成和理论计算 得到, 他们的坐标和各向同性温度因子参与结构计算,
不参与修正. 分子空间结构见图 2.

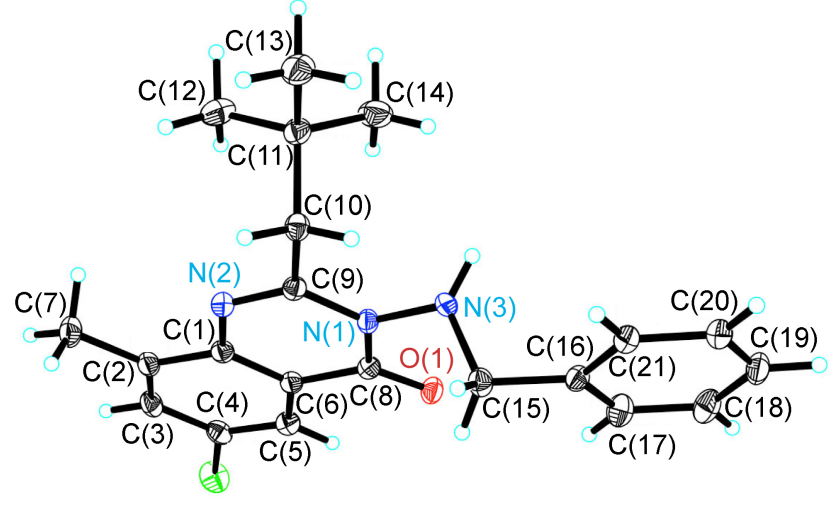

图 2 6-氯-3-芐氨基-2-新戊基-8-甲基喹唑啉-4(3H)-酮分子结 构图

Figure 2 Structure of 6-chloro-3-benzylamino-2-neopentyl8-methylquinazolin-4(3H)-one

从单晶数据可以得出 $\mathrm{N}(2)-\mathrm{C}(1), \mathrm{N}(2)-\mathrm{C}(9)$, $\mathrm{N}(1)-\mathrm{C}(9)$ 键长分别为: 1.381(3)，1.300(3)，1.393(3) A, $\mathrm{N}(2)-\mathrm{C}(9)$ 之间的键长明显小于其余二者, 说明 $\mathrm{N}(2)-$ $\mathrm{C}(9)$ 之间是碳氮双键, $N(1)-\mathrm{C}(9)-\mathrm{C}(10)-\mathrm{C}(11)$ 的扭转 角为 $95.4(3)^{\circ}, \mathrm{C}(8)-\mathrm{N}(1)-\mathrm{N}(3)-\mathrm{C}(15)$ 的扭转角为 $-73.2(3)^{\circ}$, 说明苠胺基团中的亚甲基和叔丁基中的仲 季碳原子分别位于嘧啶酮环的两侧, 以降低两个大体积 基团相互靠近所产生的势能，以形成稳定的单晶结构.

\section{References}

[1] Chen, H.-J.; Jiang, Y.-L.; Lin, C.-M. Int. J. Oncol. 2013, 43, 141.

[2] Patel, M. B.; Kumar, S. P.; Valand, N. N. J. Mol. Model. 2013, 19, 3201.

[3] Chao, Q.; Deng, L.; Shih, H. J. Med. Chem. 1999, 42, 3860.

[4] Beaulieu, P. L.; Coulombe, R.; Duan, J. Bioorg. Med. Chem. Lett. 2013, 23, 4132.

[5] Sahoo, B. M.; Dinda, S. C.; Kumar, B. V. V. R. Int. J. Pharm. Sci. Nanotechnol. 2013, 6, 2046.

[6] Abou-Seri, S. M.; Abouzid, K.; Abou El Ella, D. A. Eur. J. Med. Chem. 2011, 46, 647.

[7] Antonelli, A.; Fallahi, P.; Ferrari, S. M. Curr. Genomics 2011, 12, 626.

[8] Morgensztern, D.; Govindan, R. Chemother. Source Book, 4th ed., 2008, p. 191.

[9] (a) Zhou, J.; Fang, J. J. Org. Chem. 2011, 76, 7730.

(b) Sim, Y.-L.; Omer, N.; Khan, M. N. Tetrahedron 2013, 69, 2524.

(c) Chen, Y.; Shan, W.; Lei, M. Tetrahedron Lett. 2012, 53, 5923.

(d) Badri, R.; Alizadeh-Haddad, A.; Adlu, M. Bull. Chem. Soc. Ethiop. 2013, 27, 131.

[10] (a) Gao, X.; Cai, X.; Yan, K. Chin. J. Org. Chem. 2008, 28, 1785 (in Chinese).

(高兴文, 蔡学建, 严凯, 有机化学, 2008, 28, 1785.)

(b) Gao, X.; Cai, X.; Yan, K. Molecules 2007, 12, 2621.

[11] Xie, M.; Chen, G.; Zou, L. Chem. Res. Appl. 2010, 864 (in Chinese).

(谢敏, 陈广民, 邹丽娟, 化学研究与应用, 2010, 864.)

[12] (a) Wang, X.; Li, P.; Li, Z. J. Agric. Food Chem. 2013, 61, 9575. 
(b) Nanda, A. K.; Ganguli, S.; Chakraborty, R. Molecules 2007, 12, 2413.

(c) Ouyang, G.; Zhang, P.; Xu, G. Molecules 2006, 11, 383.

[13] (a) Liu, J.-F. Curr. Org. Synth. 2007, 4, 223.

(b) Besson, T.; Chosson, E. Comb. Chem. High Throughput Screening 2007, 10, 903.

(c) El-Mekabaty, A. Int. J. Mod. Org. Chem. 2013, 2, 81.

[14] Patil, D. A.; Patil, P. O.; Patil, G. B. Mini-Rev. Med. Chem. 2011, $11,633$.

[15] (a) Salehi, P.; Dabiri, M.; Zolfigol, M. A. Tetrahedron Lett. 2005, 41,7051 .

(b) Baghbanzadeh, M.; Dabiri, M.; Salehi, P. Heterocycles 2008, 11, 2809.

(c) Chen, J.; Wu, D.; He, F. Tetrahedron Lett. 2008, 23, 3814.

(d) Ishikawa, K.; Hosoe, T.; Itabashi, T. Sci. Pharm. 2011, 79, 937.

[16] O'Neil, M. J. The Merck Index: An Encyclopedia of Chemicals,
Drugs, and Biologicals, 14th ed., Merck \& Co., Inc., New Jersey, 2006, Monograph Number: 1658, 2653, 5691 and 8680.

[17] (a) Sati, N.; Kumar, S.; Rawat, M. S. M. Indian J. Pharm. Sci. 2009, 71, 572 .

(b) Tseng, M.-C.; Chu, Y.-H. Tetrahedron 2008, 64, 9515.

[18] (a) Nerkar, A. G.; Kudale, S. A.; Joshi, P. P. Int. J. Pharm. Pharm. Sci. 2012, 4, 449.

(b) Chikhale, H.; Lade, K.; Joshi, P. Int. J. Pharm. Pharm. Sci. 2012, 4, 466.

[19] Armarego, W. L. F.; Chai, C. L. L. Purification of Laboratory Chemicals, 5th ed., Butterworth Heinemann Press, London, 2003, p. 361, p. 378.

[20] Muller, P.; Herbst, I. R.; Spek, A. L.; Schneider, T. R.; Sawaya, M. R. Crystal Structure Refinement-A Crystallographer's Guide to SHELXL, Oxford University Press, New York, 2006.

(Qin, X.) 